\title{
A DNS study of physical mechanisms associated with influence of density ratio on turbulent burning velocity in premixed flames
}

\author{
Andrei N. Lipatnikov ${ }^{\mathrm{a}}$, Jerzy Chomiak ${ }^{\mathrm{a}}$, Vladimir A. Sabelnikov ${ }^{\mathrm{b}}$, \\ Shinnosuke Nishiki ${ }^{\mathrm{d}}$, Tatsuya Hasegawa ${ }^{\mathrm{e}}$ \\ ${ }^{a}$ Department of Applied Mechanics, Chalmers University of Technology, Göteborg, 412 96, Sweden \\ b ONERA - The French Aerospace Lab., F-91761 Palaiseau, France \\ ${ }^{\mathrm{c}}$ Department of Mechanical Engineering, Kagoshima University, Kagoshima 890-0065, Japan \\ ${ }^{\mathrm{d}}$ Institute of Materials and Systems for Sustainability, Nagoya University, Nagoya 464-8603, Japan
}

\begin{abstract}
Data obtained in 3D direct numerical simulations of statistically planar, 1D weakly turbulent flames characterized by different density ratios $\sigma$ are analyzed in order to study the influence of thermal expansion on flame surface area and turbulent burning rate. Obtained results show that, on the one hand, pressure gradient induced within the flame brush due to heat release in flamelets significantly accelerates unburned gas that deeply intrudes into combustion products in a form of an unburned mixture finger, thus, causing large-scale oscillations of the turbulent burning rate and flame brush thickness. Under conditions of the present simulations, contribution of this mechanism to creation of flame surface area is substantial and is increased by the density ratio, thus, implying an increase in the burning rate by $\sigma$. On the other hand, the total flame surface areas simulated at $\sigma=7.53$ and 2.5 are approximately equal to one another. Apparent inconsistency between these results implies existence of another thermal expansion effect that reduces the influence of the density ratio on the flame surface area and burning rate. Investigation of the issue shows that the axial flow acceleration by the combustion-induced pressure gradient not only straightforwardly creates flame surface area by pushing a finger tip into products, but also mitigates wrinkling of the flame surface (the side surface of the finger) by turbulent eddies. The latter effect is attributed to a high-speed (at $\sigma=7.53$ ) axial
\end{abstract}


flow (a jet) of unburned gas, which is induced by the axial pressure gradient within the flame brush (and the finger). This axial flow acceleration reduces a residence time of a turbulent eddy in an unburned zone of the flame brush (e.g. within the finger). Therefore, the capability of the eddy for wrinkling the flamelet surface (e.g. the side finger surface) is weakened due to a shorter residence time. Keywords: premixed turbulent flame, density ratio, Darrieus-Landau mechanism, DNS, modeling

\section{Introduction}

In spite of avalanche of papers aiming at understanding fundamentals of premixed turbulent combustion, influence of a ratio $\sigma=\rho_{u} / \rho_{b}$ of the densities of unburned and burned mixtures on turbulent flame speed $S_{t}$ is still a controversial issue. On the one hand, neither approximations of the most extensive experimental databases $[1,2,3,4,5]$ nor a few target-directed experimental studies $[6,7]$ (i.e. studies in that $\sigma$ was substantially changed by retaining approximately the same laminar flame speed $S_{L}$ ) indicate a significant effect of $\sigma$ on $S_{t}$. On the other hand, starting from the seminal work by Karlovitz et al. [8] and Scurlock and Grover [9] who put forward a concept of flame-generated turbulence, a number of models that allowed for a substantial effect of $\sigma$ on $S_{t}$ were developed, as reviewed elsewhere $[10,11]$. In particular, eventual influence of the hydrodynamic instability of thin inherently laminar flame fronts, which was independently predicted by Darrieus [12] and Landau [13], on premixed turbulent burning was addressed in a number of theoretical $[14,15,16,17,18,19,20]$, numerical $[21,22,23,24,25,26,27]$, and experimental studies $[28,29,30,31]$, as well as in review papers $[10,11,32]$ and books $[33,34,35]$. While the opposite standpoints regarding a role played by the Darrieus-Landau (DL) instability in moderately or highly turbulent flames can be found in the literature, this phenomenon is commonly considered to increase burning rate at least in weakly turbulent flames where the rms turbulent velocity $u^{\prime}$ is on the order of or less than $S_{L}$. The major goal of the present work is to discuss Direct Numerical Simulation (DNS) data that imply that a physical 
mechanism that causes the DL instability (i.e. the influence of combustion-induced pressure gradient on the unburned gas flow) not only straightforwardly increases $S_{t}$, but also can act in the opposite direction by mitigating turbulent wrinkling of the instantaneous flame surface at $u^{\prime} / S_{L}=O(1)$.

Because the latter phenomenon was found in attempts to explain an apparent inconsistency between DNS results, a large part of the present paper will be devoted to showing this apparent inconsistency and considering various effects that might be relevant to explaining it. In the next section, the attributes of the DNS database are summarized. The apparent inconsistency between numerical results, various relevant effects, and, in particular, mitigation of turbulent wrinkling of the instantaneous flame surface due to acceleration of the unburned gas flow by the combustion-induced pressure gradient are discussed in the third section followed by conclusions.

\section{DNS Database}

Because DNSs addressed here were discussed in detail elsewhere [36, 37] and the computed data were used in several papers $[38,39,40,41,42,43,44,45,46,47,48,49,50]$, we will restrict ourselves to a brief summary of these compressible simulations.

The DNSs dealt with statistically planar, 1D, adiabatic flames modeled by unsteady 3D continuity, Navier-Stokes, and energy equations, as well as a transport equation for the mass fraction $Y$ of a deficient reactant and the ideal gas state equation. The Lewis and Prandtl numbers were equal to 1.0 and 0.7, respectively. Combustion chemistry was reduced to a single reaction. Temperature-dependence of molecular transport coefficients was taken into account, e.g. $\nu=\nu_{u}\left(T / T_{u}\right)^{0.7}$, where $\nu$ is the kinematic viscosity of the mixture, $T$ is the temperature, and subscript $u$ designates unburned gas.

The computational domain was a rectangular box $\Lambda_{x} \times \Lambda_{y} \times \Lambda_{z}$, with $\Lambda_{x}=8 \mathrm{~mm}, \Lambda_{y}=\Lambda_{z}=4 \mathrm{~mm}$, and was resolved using a uniform mesh of $512 \times 128 \times 128$ points. Homogeneous isotropic turbulence $\left(u^{\prime}=0.53 \mathrm{~m} / \mathrm{s}\right.$, integral length scale $L=3.5 \mathrm{~mm}$, Kolmogorov length scale $\eta=0.14 \mathrm{~mm}$, and the turbulent Reynolds number $\left.R e_{t}=u^{\prime} L / \nu_{u}=96[36,37]\right)$ was generated in a separate box and 
Table 1: Flame characteristics

\begin{tabular}{ccc} 
& Case H & Case L \\
\hline$\sigma=\rho_{u} / \rho_{b}$ & 7.53 & 2.5 \\
$S_{L}, \mathrm{~m} / \mathrm{s}$ & 0.600 & 0.416 \\
$\delta_{L}, \mathrm{~mm}$ & 0.217 & 0.158 \\
$U\left(t_{2} \leq t\right), \mathrm{m} / \mathrm{s}$ & 1.15 & 0.79 \\
\hline
\end{tabular}

was injected into the computational domain through the left boundary $x=0$. In the computational domain, the turbulence decayed along the direction $x$ of the mean flow. The flow was periodic in $y$ and $z$ directions.

At $t=0$, a planar laminar flame was embedded into statistically the same turbulence assigned for the velocity field in the entire computational domain. Subsequently, the inflow velocity was increased twice, i.e. $U\left(0 \leq t<t_{1}\right)=S_{L}<U\left(t_{1} \leq t<t_{2}\right)<U\left(t_{2} \leq t\right)$, in order to keep the flame in the computational domain till the end $t_{3}$ of simulations.

Three cases H, M, and L characterized by High, Medium, and Low, respectively, density ratios $\sigma=\rho_{u} / \rho_{b}$ were studied $[36,37]$. Here, we restrict ourselves to cases $\mathrm{H}$ and L associated with the highest and lowest $\sigma$, respectively. It is worth stressing that even case $\mathrm{L}$ is far from a particular case of $\sigma=1$ (a constant density).

Characteristics of flames $\mathrm{H}$ and $\mathrm{L}$ are listed in Table 1 , where $\delta_{L}=\left(T_{b}-T_{u}\right) / \max |d T / d x|$ is the laminar flame thickness and subscript $b$ designates burned gas. The laminar flame speeds or thicknesses are different in these cases, because the same molecular diffusivity $D_{u}$ of the deficient reactant in unburned mixture and the same expression for the mass reaction rate $W_{c}(Y, T)$ were used independently of the density ratio.

Results reported later were obtained for $t_{2} \leq t \leq t_{3}$, with the instants $t_{2}$ or $t_{3}$ being different in cases $\mathrm{H}$ and $\mathrm{L}$. Because the mean inlet velocity was constant at $t \geq t_{2}$, see $U\left(t_{2} \leq t\right)$ in Table 1 , no external acceleration affected results obtained in the laboratory coordinate framework.

When processing the DNS data, we evaluated both time-dependent mean quantities, which were 
averaged over transverse $y z$-planes at various instants, and mean quantities, which were averaged not only over transverse $y z$-planes, but also over time interval on the order of $10 \mathrm{~ms}$ (about 200 snapshots). In the rest of the present paper, the fully mean and time-dependent average values of a quantity $q$ will be designated using overbars and angular brackets, respectively, i.e. $\bar{q}(x)$ and $\langle q\rangle(x, t)$, respectively. Moreover, we determined joint Probability Density Functions (PDFs) $P(q, c, x)$ for various flame characteristics $q(\mathbf{x}, t)$, the combustion progress variable $c(\mathbf{x}, t)=\left[T(\mathbf{x}, t)-T_{u}\right] /\left(T_{b}-T_{u}\right)$, which was also equal to $1-Y(\mathbf{x}, t) / Y_{u}$ under conditions of the present study $(L e=1)$, and the axial coordinate $x$. When determining such PDFs, an interval of $0 \leq c \leq 1$ was divided in 100 sub-intervals. Subsequently, these PDFs and dependencies $\bar{q}(x)$ were transformed to $P(q, c, \bar{c})$ and $\bar{q}(\bar{c})$, respectively, using profiles of $\bar{c}(x)$, computed by processing the same DNS data.

Quantities $\bar{q}_{u}(\bar{c})$ conditioned to unburned gas and conditioned joint PDFs $P_{u}(q, \bar{c})$ were computed by averaging over both transverse planes and time and considering only points $\mathbf{x}$ such that $c(\mathbf{x}, t)<\varepsilon \ll 1$. Results reported in the following were obtained for $\varepsilon=0.005$ if the opposite is not specified, with weak sensitivity of the conditioned data to variations in $\varepsilon$ from 0.001 to 0.1 being checked. Conditioned quantities $\langle q \mid \xi\rangle$ were evaluated for $\xi-0.005<c(\mathbf{x}, t) \leq \xi+0.005$ and were averaged over both transverse planes and time. Henceforth, such a conditioned quantity is designated with $\langle q \mid c\rangle$. In particular, $\langle q \mid c=0.85\rangle$ is the value of $q$ conditioned to the reaction zone, where $W_{c}(c)$ is close to its peak value $W_{c, \max }$.

\section{Results and Discussion}

The structure of this section is as follows. In Sect. 3.1, we briefly remind certain results reported by us earlier [47] and supplement them with new figures. Such a reminder is required in order to show an effect (Sect. 3.2) to be explained in Sects. 3.3-3.6. Relevant issues are discussed in Sects. 3.7 and 3.8 . 
(a) case $\mathrm{H}$

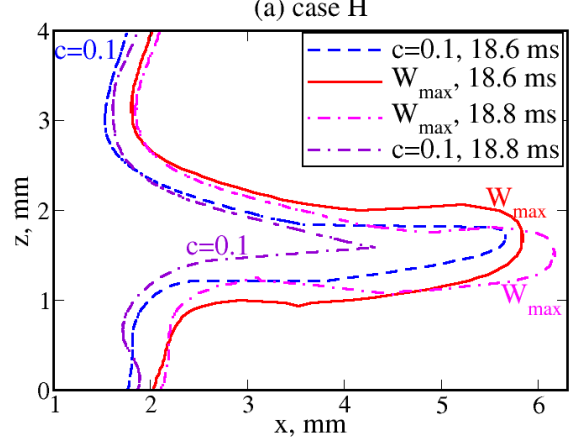

(b) case L

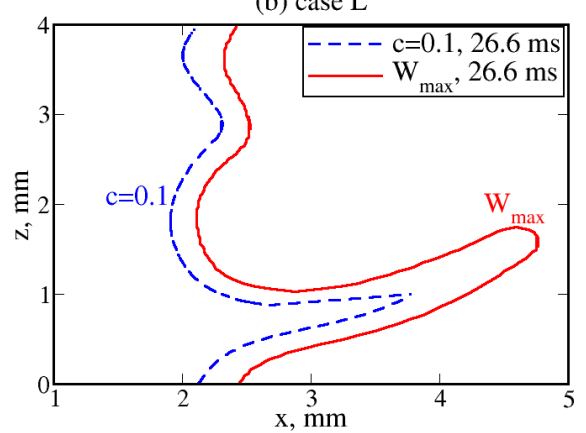

Figure 1: Iso-lines $c\left(x, y_{f t}, z, t\right)=0.1$ and $W_{c}\left(x, y_{f t}, z, t\right)=W_{c, m}$ computed (a) in case $\mathrm{H}$ at $t=18.6$ and 18.8 ms or (b) in case $\mathrm{L}$ at $t=26.6 \mathrm{~ms}$

\subsection{Fingering instability driven by combustion-induced pressure gradient}

By analyzing the DNS data by Nishiki et al. [36, 37], we recently studied the so-called Unburned Mixture Fingers (UMFs), see Fig. 1, that deeply intrude into combustion products, thus, increasing instantaneous total flame surface area $\langle A\rangle(t)$, turbulent burning velocity $\left\langle U_{t}\right\rangle(t)$, and mean flame brush thickness. Here, both ( $\mathrm{H}$ and $\mathrm{L})$ flames propagate from right to left, the coordinates $y_{f t}(t)$ and $z_{f t}(t)$ of the finger tip are the $y$ and $z$ coordinates, respectively, of a point $x_{f t}(t)=x\left[y_{f t}(t), z_{f t}(t), t\right]$ that is most close to the right boundary among all points associated with a local peak $W_{c, m}$ of the mass reaction rate $W_{c}$ vs. $x$,

Figure 2a shows large-scale oscillations of $\left\langle U_{t}\right\rangle(t) / S_{L}$ and $\langle A\rangle(t)$, see solid and dotted lines, respectively, and Fig. 2b shows large-scale oscillations of a mean flame brush thickness $\left\langle\delta_{t, 1}\right\rangle(t)$ or $\left\langle\delta_{t, 2}\right\rangle(t)$, see solid and dashed lines, respectively. As discussed in detail elsewhere [47] these oscillations are controlled by growth and disappearance of UMFs. Here,

$$
\begin{gathered}
\left\langle U_{t}\right\rangle(t)=\int_{0}^{\Lambda_{x}} \int_{0}^{\Lambda_{y}} \int_{0}^{\Lambda_{z}} \frac{W_{c}(\mathbf{x}, t) d x d y d z}{\rho_{u} \Lambda_{y} \Lambda_{z}} \\
\langle A\rangle(t)=\int_{0}^{\Lambda_{x}} \int_{0}^{\Lambda_{y}} \int_{0}^{\Lambda_{z}} \frac{|\nabla c|(\mathbf{x}, t) d x d y d z}{\Lambda_{y} \Lambda_{z}} \\
\left\langle\delta_{t, 1}\right\rangle(t)=\frac{1}{\max \{\partial\langle c\rangle / \partial x\}}
\end{gathered}
$$

and $\left\langle\delta_{t, 2}\right\rangle(t)$ is equal to a distance between surfaces $x=x_{1}(t)$ and $x=x_{2}(t)$ associated with $\langle c\rangle\left(x_{1}, t\right)=$ 

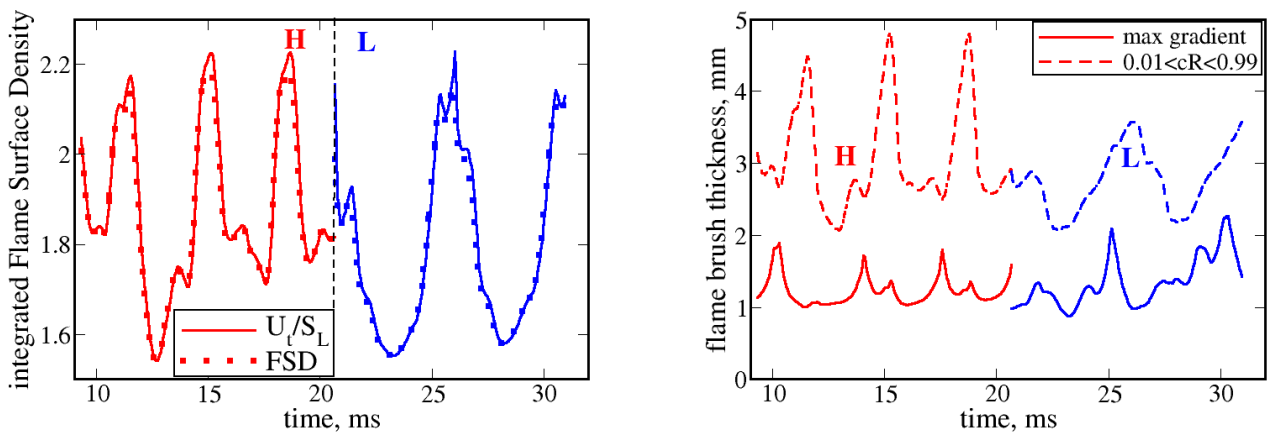

Figure 2: Oscillations of (a) $\left\langle U_{t}\right\rangle / S_{L}$ (solid lines) and $\langle A\rangle$ (dotted lines) and (b) mean flame brush thicknesses, obtained in cases $\mathrm{H}$ (red lines, $9.3<t<20.7 \mathrm{~ms}$ ) and $\mathrm{L}$ (blue lines, $20.6<t<31 \mathrm{~ms}$ ).

0.01 and $\langle c\rangle\left(x_{2}, t\right)=0.99$, respectively. It is worth noting that oscillations of $\left\langle U_{t}\right\rangle(t)$ with a significantly higher (when compared to Fig. 2a) relative amplitude were obtained in recent DNS by Poludnenko $[51]$.

Local structures that look similar to two UMFs shown in Fig. 1 can be identified in numerous experimental (e.g. see Fig. 2 [52], Fig. 1 [53], Figs. 9 and 10 [54], Fig. 10 [55], Fig. 4 [56], Figs. 3, 7, and 12 [57], Fig. 4d [58], Fig. 4 [59], Figs. 2 and 4 [60], Figs. 4a and 10 [61], Figs. 6 and 16 [62], Fig. 4 [63], Figs. 6, 8-10 [64]) and numerical (e.g. Fig. 1 [26], see Fig. 4 [65], Fig. 3 [66], Fig. 10 [67], Fig. 5 [68], Fig. 8 [69], Fig. 4d [70], Fig. 4a [71]) images.

Physical mechanisms that control the growth of UMFs were explored in recent DNS studies [47, 51]. In particular, by analyzing the present DNS data, we have shown that the simulated finger growth is controlled by significant acceleration of unburned gas by normal (to the mean flame brush) pressure gradient induced due to combustion in surrounding flamelets ${ }^{1}$ [47]. Such a flow acceleration is clearly seen in Fig. 3, which reports the axial profiles of the instantaneous axial flow velocity $u\left[x, y_{f t}\left(t_{m}\right), z_{f t}\left(t_{m}\right), t_{m}\right]$, computed along the axis $\left\{y=y_{f t}\left(t_{m}\right), z=z_{f t}\left(t_{m}\right)\right\}$ of an UMF in case $\mathrm{H}$ at three instants $t=t_{m}$. Besides a sharp increase in $u(x)$ downstream of point $\mathrm{A}, \mathrm{B}$, or $\mathrm{C}$, which is controlled by the local density decrease at the finger tip, the axial velocity grows even in the unburned

\footnotetext{
${ }^{1}$ As discussed in detail elsewhere [46], the present DNSs address the flamelet regime of premixed turbulent combustion.
} 


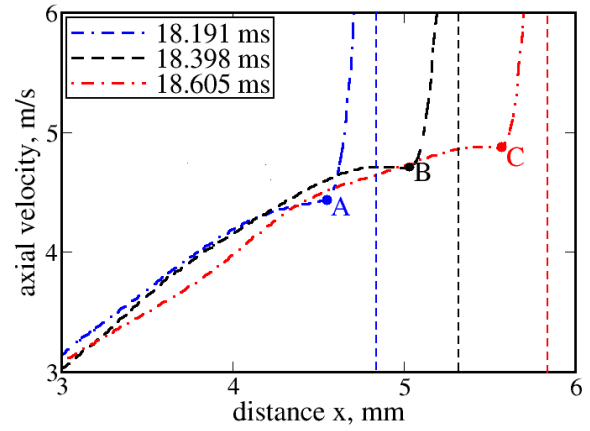

Figure 3: Growth of the axial flow velocity along the axis $\left\{y=y_{f t}\left(t_{m}\right), z=z_{f t}\left(t_{m}\right)\right\}$ of an UMF in case $\mathrm{H}$ at various instants $t=t_{m}$ specified in legends. Vertical dashed lines show $x$ associated with the peak reaction rate along the finger axis at the instants $t_{m}$.

gas, i.e. upstream of point $\mathrm{A}, \mathrm{B}$, or $\mathrm{C}$, with the peak values of the axial unburned gas velocity (see points A-C) being higher by a factor of about four ${ }^{2}$ than the inlet velocity (and an order of magnitude higher than the inlet $u^{\prime}$ ).

Such a strong acceleration of the constant-density flow of unburned gas cannot be driven by weak turbulence. In order to reveal a physical mechanism that controls such a strong increase in the axial velocity of the unburned gas, we computed terms $T_{n}(\mathbf{x}, t)$ in the following balance equation for the axial momentum

$$
\frac{\partial u}{\partial t} \underbrace{+u \frac{\partial u}{\partial x}}_{T_{1}} \underbrace{+v \frac{\partial u}{\partial y}+w \frac{\partial u}{\partial z}}_{T_{2}}=\underbrace{-\frac{1}{\rho} \frac{\partial p}{\partial x}}_{T_{3}}+\underbrace{\frac{1}{\rho} \frac{\partial \tau_{x l}}{\partial x_{l}}}_{T_{4}},
$$

conditioned to $c(\mathbf{x}, t)<0.005$. Here,

$$
\tau_{i j}=\rho \nu\left(\frac{\partial u_{i}}{\partial x_{j}}+\frac{\partial u_{j}}{\partial x_{i}}-\frac{2}{3} \delta_{i j} \frac{\partial u_{l}}{\partial x_{l}}\right)
$$

is the viscous stress tensor, $\delta_{i j}$ is the Kronecker delta, and the summation convention applies for the repeated index $l$. Obtained results, which are discussed in detail elsewhere [47], show that the axial reactant acceleration is controlled by term $T_{3}$, with this pressure-gradient term dominating in the

\footnotetext{
${ }^{2}$ It is worth noting that, under conditions of the present study, the axial acceleration of lighter combustion products is even stronger and results in $\bar{u}_{b}(x)>\bar{u}_{u}(x)$ and the countergradient transport of $c$, as discussed in detail elsewhere [37, 45].
} 


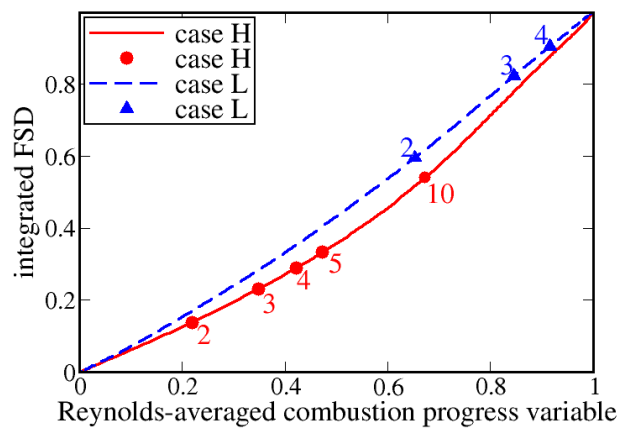

Figure 4: Relative flame surface area $\bar{A}(\bar{c}) / \bar{A}(1)$ (lines) and values $c_{\beta}$ (symbols) of the lowest $\bar{c}$ such that the probability $\mathcal{P}_{u}(\psi \geq$ $\beta, \bar{c}) \geq 0.5$. Values of $\beta$ are shown near symbols.

significant part of the mean flame brush in case $\mathrm{H}$.

For instance, in order to reveal an important role played by the pressure-driven acceleration of the unburned gas, we compared local magnitudes $\left|T_{1}\right|$ and $\left|T_{3}\right|$ of the axial-acceleration and pressuregradient terms, respectively, with the local magnitudes $\left|T_{2}\right|$ and $\left|T_{4}\right|$ of two other terms. In particular, we evaluated an instantaneous ratio $\psi(\mathbf{x}, t)$ of $\min \left\{\left|T_{1}\right|,\left|T_{3}\right|\right\}$ to $\max \left\{\left|T_{2}\right|,\left|T_{4}\right|\right\}$. A large value of $\psi(\mathbf{x}, t)$ indicates that the local magnitudes of both terms $T_{1}$ and $T_{3}$ are large when compared to the local magnitudes of terms $T_{2}$ and $T_{4}$, i.e. the axial flow acceleration is controlled by the axial pressure gradient, whereas the local contribution of turbulence (terms $T_{2}$ and $T_{4}$ ) to the acceleration is weak. Accordingly, selection of regions characterized by a large $\psi(\mathbf{x}, t)$ allows us to find regions where the axial flow is driven by the axial pressure gradient. For instance, abscissa coordinates of symbols plotted in Fig. 4 are equal to values $c_{\beta}$ of the lowest mean $\bar{c}$ such that probability $\mathcal{P}_{u}(\psi \geq \beta, \bar{c})=\int_{\beta}^{\infty} P(\psi, c<$ $0.005, \bar{c}) d \psi$ of finding large $\psi(\mathbf{x}, t)>\beta$, conditioned to unburned gas $(c<0.005)$, is $50 \%$ or more, with this probability being larger than $50 \%$ if $\bar{c}>c_{\beta}$. Thus, if $\beta$ is large, then, the pressure-driven axial acceleration dominates at $\bar{c}>c_{\beta}$, i.e. to the right of symbols in Fig. 4 .

In order to assess contributions of such regions to creation of flame surface area, we also evaluated the following integral

$$
\bar{A}(x)=\int_{t_{2}}^{t_{3}} \int_{0}^{x} \int_{0}^{\Lambda_{y}} \int_{0}^{\Lambda_{z}} \frac{|\nabla c|(\mathbf{x}, t)}{\left(t_{3}-t_{2}\right) \Lambda_{y} \Lambda_{z}} d t d x d y d z
$$


which characterizes the mean flame surface area available upstream of a plane $x=$ const. Subsequently, the obtained dependencies $\bar{A}(x)$ were converted to $\bar{A}(\bar{c})$ and a ratio of $\bar{A}\left(\bar{c}_{*}\right) / \bar{A}(1)$ was used to assess contribution of a region of $\bar{c} \leq \bar{c}_{*}$ to production of the total flame surface area. Results are shown in lines in Fig. 4. It is worth stressing that these results are time-averaged, i.e. they are averaged not only over time intervals associated with appearance of elongated UMFs, e.g. see local peaks of $\left\langle\delta_{t, 2}\right\rangle(t)$-curves in Fig. 2b, but also over time intervals during that UMFs vanished.

Circles on the solid line $(\sigma=7.53$, case $\mathrm{H})$ indicate a significant contribution to the total flame surface area from zones where turbulence (terms $T_{2}$ and $T_{4}$ ) plays a minor role in Eq. (4) conditioned to unburned gas when compared to the axial pressure gradient. Indeed, within a zone of $\bar{c} \geq c_{10} \approx 0.67$ (the right red circle), (i) about $50 \%$ of the total flame surface area is found and (ii) the local magnitudes of the axial convection and pressure gradient terms $T_{1}$ and $T_{3}$, respectively, are larger than the local magnitudes of the transverse convection and viscous terms $T_{2}$ and $T_{4}$, respectively, by a factor of 10 or more with probability of $50 \%$ or higher.

Thus, Fig. 1a and red circles and solid line in Fig. 4 imply that the axial acceleration of the unburned gas by the combustion-induced axial pressure gradient contributes significantly to production of flame surface area. In the following, this physical mechanism will be called DL mechanism, because generally the same physical mechanism causes the DL instability of laminar premixed flames, i.e. both phenomena are driven by pressure perturbations in unburned gas due to thermal expansion. Nevertheless, it is worth stressing that there are no cogent reasons to reduce the growth of an UMF to development of the DL instability. In other words, both phenomena (the DL instability and UMFs) appear to be two manifestations of the same governing mechanism. Because the former manifestation of this mechanism was predicted by Darrieus [12] and Landau [13], we use a term "DL mechanism" in the present paper, but the term is solely used in this sense. 


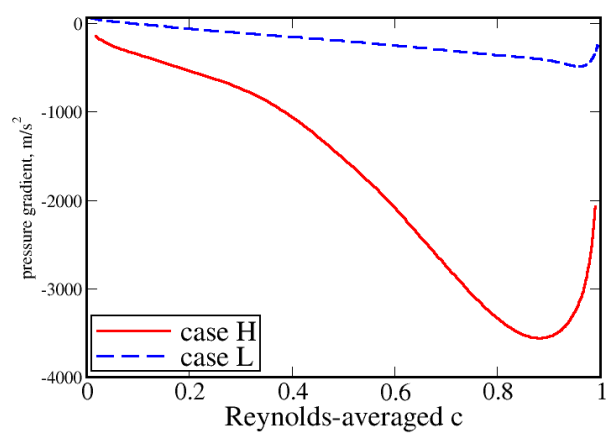

Figure 5: Axial pressure gradient $\rho_{u}^{-1}(\overline{\partial p / \partial x})_{u}$ averaged in unburned gas over transverse planes and time vs. the Reynoldsaveraged combustion progress variable $\bar{c}$.

\subsection{Issue to be addressed}

Because phenomena discussed in the previous subsection are driven by the combustion-induced axial pressure gradient in unburned gas and the magnitude of this pressure gradient is decreased when the density ratio is decreased, see Fig. 5, we could expect that these phenomena are significantly less pronounced in case L when compared to case H. Indeed, certain aforementioned effects are mitigated by a decrease in $\sigma$. For instance, (i) H-finger is longer than L-finger, cf. Figs. 1a and 1b, and (ii) the peak thickness $\left\langle\delta_{t, 2}\right\rangle(t)$ is larger in case $\mathrm{H}$ when compared to case $\mathrm{L}$, cf. red and blue dashed lines in Fig. 2b.

For the goals of the present work, the following difference between cases $\mathrm{H}$ and $\mathrm{L}$ is of paramount importance. The left circle on a solid line in Fig. 4 shows that, if $\beta=2$ in case $\mathrm{H}$, then, $c_{\beta} \approx 0.22$ and more than $86 \%$ of the total flame surface area is localized to a region where the probability $\mathcal{P}_{u}(\psi \geq 2) \geq 0.5$. To the contrary, in case $\mathrm{L}, c_{2} \approx 0.65$ is significantly larger and only about $40 \%$ of the total flame surface area is localized to a region associated with $\mathcal{P}_{u}(\psi \geq 2) \geq 0.5$. Furthermore, if $\sigma=2.5$ and $\beta=4$, then, only $10 \%$ of the total flame surface area are associated with $\bar{c}>c_{4} \approx 0.92$, see the right triangle on the dashed line, whereas about $70 \%$ of the total flame surface area are associated with $\bar{c}>c_{4}$ in case $\mathrm{H}$.

These results indicate that the contribution of the DL mechanism to production of flame surface 
area is substantially stronger in case $\mathrm{H}$ when compared to case $\mathrm{L}$, thus, implying that the time-averaged ratio of $\bar{U}_{t} / S_{L}$ should be significantly larger in the former case. However, red and blue lines in Fig. 2a show that both mean and peak values of both $\langle A\rangle(t)$ and $\left\langle U_{t}\right\rangle(t) / S_{L}$ are comparable in cases $\mathrm{H}$ and $\mathrm{L}$. Moreover, $\bar{U}_{t} / S_{L}$ extracted from the DNS data are almost equal, i.e. 1.88 and 1.77 in cases $\mathrm{H}$ and $\mathrm{L}$, respectively.

Thus, at first glance, Figs. 2 and 4 appear to be inconsistent with one another, because the former (latter) figure implies that the density ratio weakly (strongly) affects the total flame surface area. The rest of the paper aims at clarifying this apparent paradox and revealing a physical mechanism that counterbalances a stronger (in case $\mathrm{H}$ ) increase in the total flame surface area due to the DL mechanism, as indicated in Fig. 4.

To do so, in the next subsections, we (i) step-by-step consider differences between cases $\mathrm{H}$ and L that might be of importance in order to explain the discussed paradox, (ii) reject differences that do not explain it (Sects. 3.3-3.5), and (iii) highlight a physical mechanism that appears to explain the paradox (Sect. 3.6). Finally, we discuss some relevant issues in Sects. 3.7 and 3.8. It is worth stressing that solely physical mechanisms that could stronger contribute to production of flame surface area in case $\mathrm{L}$ when compared to case $\mathrm{H}$ are addressed in the rest of the present section, because solely such mechanisms appear to be relevant to explaining the paradox referred to.

\subsection{Turbulence in unburned gas}

Flames $\mathrm{H}$ and $\mathrm{L}$ are characterized not only by significantly different density ratios, but also by different $S_{L}$. As a result, the two flames experience different turbulence. Indeed, the rates of the turbulence decay in the axial direction are different due to the difference in the mean inlet axial velocities $U\left(t>t_{2}\right)$, see Table 1 , which are set approximately equal to $\bar{U}_{t}$ in order to keep the flame brush within the computational domain in each case. Due to a higher $S_{L}$ in case $\mathrm{H}$ when compared to $\mathrm{L}$, in the former case, $\bar{U}_{t}$ and, hence, $U\left(t>t_{2}\right)$ are larger and the turbulence decay in the axial direction is less pronounced due to a shorter residence time required for a fluid particle to move from 
(a)

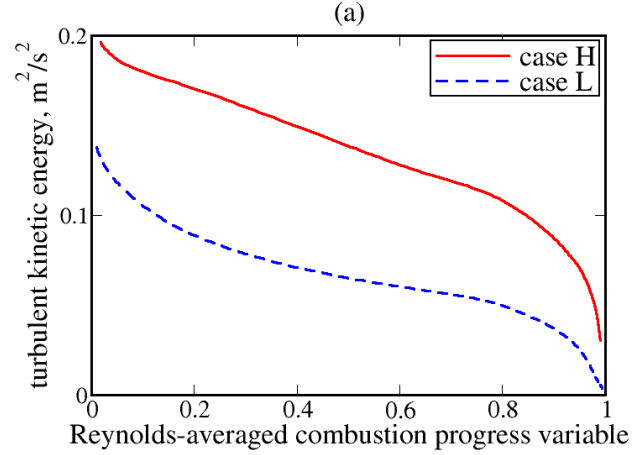

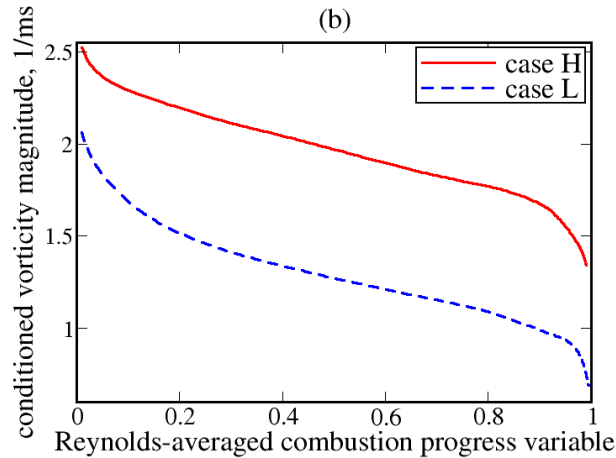

Figure 6: Turbulent kinetic energy $\overline{u_{k}^{\prime} u_{k}^{\prime}} / 2$ and magnitude $\overline{\left(\omega_{k} \omega_{k}\right)^{1 / 2}}$ of vorticity vector $\vec{\omega}=\nabla \times \mathbf{u}$, averaged over transverse planes and time in unburned gas, vs. the Reynolds-averaged combustion progress variable $\bar{c}$.

the left boundary to the flame brush. For instance, the rms turbulent velocity averaged over plane $\bar{c}=0.01$ is equal to 0.36 or $0.29 \mathrm{~m} / \mathrm{s}$ in case $\mathrm{H}$ or $\mathrm{L}$, respectively.

Figure 6 shows that conditioned turbulent kinetic energy and magnitude of vorticity vector, which characterize large and small-scale eddies, respectively, are higher in case $\mathrm{H}$ than in case L. Thus, flame $\mathrm{H}$ experiences a more intense incoming turbulence and, under such a low ratio of $u^{\prime} / S_{L}$, a more intense turbulence is commonly associated with a larger flame surface area. Therefore, the difference in the characteristics of the incoming turbulence experienced by flame $\mathrm{H}$ or $\mathrm{L}$ does not seem to explain why the values of $\bar{U}_{t} / S_{L}$ obtained in the two cases are close to one another in spite of significantly stronger contribution of the DL mechanism to the production of the flame surface area in case $\mathrm{H}$, indicated in Fig. 4.

It is worth noting that, while $u^{\prime}$ is higher at the leading edge of the mean flame brush in case $\mathrm{H}$ when compared to case L, a ratio of this $u^{\prime}$ to $S_{L}$ shows the opposite trend. It is equal to 0.6 or 0.7 in case $\mathrm{H}$ or L, respectively, because $S_{L}$ is higher in the former case. Eventual relevance of the difference in values of $S_{L}$ to explaining the paradox in question is addressed in Sect. 3.5. 


\subsection{Turbulence within flamelets}

Because both dilatation and an increase in the local viscosity within flamelets are more pronounced in case $\mathrm{H}$ characterized by a significantly larger $\sigma$ than case $\mathrm{L}$, one may assume that, even if flame $\mathrm{H}$ experiences a more intense turbulence in the incoming unburned gas, the thin reaction zone is affected by a weaker turbulence due to a stronger dilatation and a more rapid viscous dissipation of the turbulence within flamelets in case $\mathrm{H}$ when compared to L. Accordingly, one may further assume that, in the former case, the reaction zone is less wrinkled and stretched and this effect reduces the flamelet surface area in case $\mathrm{H}$ when compared to $\mathrm{L}$, thus, counterbalancing the opposite action of the DL mechanism, shown in Fig. 4. However, such assumptions do not hold under conditions of the present DNS.

Indeed, first, it is worth remembering, that an increase in the temperature and a decrease in the density within flamelets not only cause the local turbulence decay due to dilatation and enhanced viscous dissipation, but also generate the local turbulence e.g. due to vorticity production by baroclinic torque. For instance, under conditions of the present DNS, the vorticity production by baroclinic torque within flamelets overwhelms the local vorticity decay due to dilatation (mainly) and viscous dissipation in case $\mathrm{H}$, but the net effect is opposite in case L, see Fig. 4 in [44]. As a result, vorticity magnitude conditioned to the reaction zone, i.e. $0.7<c(\mathbf{x}, t)<0.9$, is significantly larger in case $\mathrm{H}$ than in case $\mathrm{L}$ $[44]$.

Second, Fig. 7 shows that the normalized local strain rate $\left\langle\tau_{c} a_{t} \mid c\right\rangle$ conditioned to the instantaneous combustion progress variable $c$ is significantly higher at large values of the mean combustion progress variable $\bar{c}$ in case $H$, see red solid and dashed lines, when compared to case L, see blue dotted-dashed lines. Moreover, the strain rate is not reduced within flamelets at the trailing zone of the mean flame brush, see red solid and blue double-dotted-dashed lines, which show an increase in $\left\langle a_{t} \mid c\right\rangle$ by $c$ at $\bar{c}=0.9$ in both cases $\mathrm{H}$ and $\mathrm{L}$, respectively. Here, large values of $\bar{c}$ are addressed, because the axial pressure gradient dominates in Eq. (4) in that flame region, $a_{t}=\nabla \cdot \mathbf{u}-\mathbf{n n}: \nabla \mathbf{u}$ is the local strain rate, $\mathbf{u}$ is 


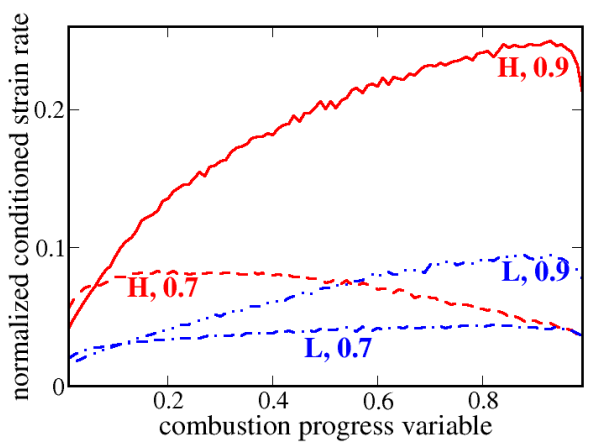

Figure 7: Normalized conditioned strain rate $\tau_{c}\left\langle a_{t} \mid c\right\rangle$ vs. $c$. The strain rate was computed for values of the Reynoldsaveraged combustion progress variable, specified near each curve. Red solid and dashed or blue dotted-dashed lines show results obtained in case $\mathrm{H}$ or $\mathrm{L}$, respectively.

velocity vector, $\mathbf{n}=-\nabla c /|\nabla c|$ is the unit vector locally normal to a flamelet, symbol $\langle q \mid c\rangle$ designates a quantity $q$ averaged at certain $c(\mathbf{x}, t)$ over transverse planes and time, i.e. $\langle q \mid c\rangle$ depends on $x$, but is independent of $t$, contrary to unconditioned time-dependent mean quantities $\langle q\rangle(x, t)$ discussed earlier, and $\tau_{c}=D_{u} / S_{L}^{2}$ is the chemical time scale. It is worth also noting that, due to a larger $S_{L}$, the time scale $\tau_{c}$ is lower by a factor of two in case $\mathrm{H}$ when compared to $\mathrm{L}$, i.e. difference in dimensional $\left\langle a_{t} \mid c\right\rangle$ is even stronger than difference in the normalized $\left\langle\tau_{c} a_{t} \mid c\right\rangle$, which is plotted in Fig. 7.

Thus, difference between turbulence characteristics conditioned to flamelets in cases $\mathrm{H}$ and $\mathrm{L}$ is not associated with a larger $\langle A\rangle(t)$ in the latter case and, therefore, cannot explain the discussed paradox.

\subsection{Flamelet propagation}

Due to difference in $S_{L}$ in cases $\mathrm{H}$ and L, self-propagation of flamelets in a turbulent flow can differently affect flame surface area in the two cases. If the local flamelet curvature $\kappa_{m}=\nabla \cdot \mathbf{n} / 2$ is positive (i.e. the curvature center is in burned gas), then, an increase in $S_{L}$ results in increasing the local stretch rate, thus, enhancing the local production of flame surface area. Such an effect makes $\langle A\rangle(t)$ larger in case $\mathrm{H}$ when compared to $\mathrm{L}$. Therefore, this effect cannot explain the paradox addressed by us and will not be discussed in the following. 
If the local flamelet curvature is negative, then, an increase in $S_{L}$ results in decreasing the local stretch rate, thus, impeding the local production of flame surface area. Moreover, an increase in $S_{L}$ is associated with enhancement of annihilation of flame surface area due to collision of flamelets [72]. The two effects might reduce $\langle A\rangle(t)$ stronger in case $\mathrm{H}$ when compared to $\mathrm{L}$ and, hence, appear to be relevant to explaining the paradox.

Accordingly, these two effects are addressed in the present subsection, whose focus is placed on large values of the mean ${ }^{3}$ combustion progress variable $\bar{c} \geq 0.5$ for the following two reasons. First, due to purely geometrical arguments, the probabilities of both (i) finding negatively curved flamelets and (ii) flamelet collisions are increased by $\bar{c}$. Consequently, the two effects should be more pronounced at large $\bar{c}$ and, therefore, the trailing part of the mean flame brush is of the most interest in order to assess whether or not a decrease in $S_{L}$ can result in increasing $\langle A\rangle(t)$. Second, as discussed earlier, the axial pressure gradient dominates in Eq. (4) also at large $\bar{c}$.

In order to assess whether or not the difference in $S_{L}$ in cases $\mathrm{H}$ and L can explain the paradox, we evaluated various terms in the following well-known transport equation for the Flame Surface Density $(\mathrm{FSD}) \Sigma \equiv|\nabla c|[72]$

$$
\frac{d}{d x} \overline{u \Sigma}=\overline{a_{t} \Sigma}-\frac{d}{d x} \overline{S_{d} n_{x} \Sigma}+\overline{S_{d} \Sigma \nabla \cdot \mathbf{n}}
$$

written in the statistically stationary, planar, $1 \mathrm{D}$ case addressed by us. Here, $n_{x}$ is the $x$-component of the unit normal vector $\mathbf{n}$ and $S_{d}=\left[\nabla \cdot(\rho D \nabla c)+W_{c}\right] /(\rho|\nabla c|)$ is the local displacement speed. Under conditions of the present DNS (weak turbulence), $S_{d}$ is expected to scale as $\rho_{u} S_{L} / \rho$ to the leading order within the largest part of the mean flame brush (with exception of its trailing edge, as discussed in Sect. 3.7).

In order to be confident in the present DNS data, we evaluated each term in this transport equation and checked that the obtained residuals were small in the entire flame brushes. These results are

\footnotetext{
${ }^{3}$ Results discussed in the previous sections were conditioned to the local combustion progress variable $c$, rather than the mean $\bar{c} \geq 0.5$.
} 


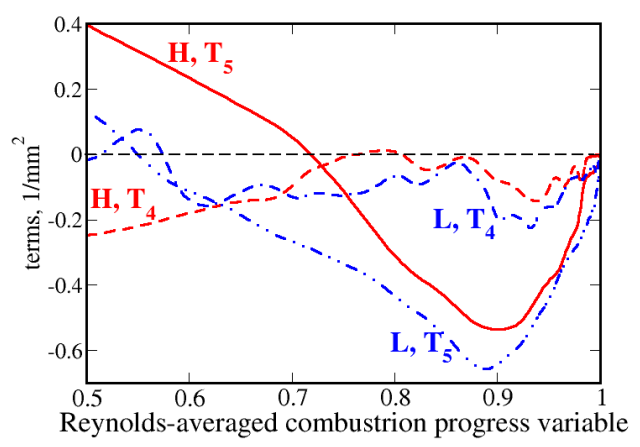

Figure 8: Terms $T_{4}$ and $T_{5}$ in Eq. (8), obtained in cases H (red solid and dashed lines) and L (blue dotted-dashed lines), vs. the Reynolds-averaged combustion progress variable.

reported elsewhere [49]. Here, we restrict ourselves solely to results that are straightforwardly relevant to the goals of the present work, i.e. explaining the paradox under discussion.

Accordingly, let us rewrite Eq. (7) as follows

$$
\begin{aligned}
\frac{d \bar{\Sigma}}{d x}= & \underbrace{-\frac{\bar{\Sigma}}{\bar{u}} \frac{d \bar{u}}{d x}}_{T_{1}} \underbrace{-\frac{1}{\bar{u}} \frac{d \overline{u^{\prime} \Sigma^{\prime}}}{d x} \underbrace{+\frac{\overline{a_{t} \Sigma}}{\bar{u}}}_{T_{3}}}_{T_{2}} \\
& \underbrace{-\frac{1}{\bar{u}} \frac{d \overline{S_{d} n_{x} \Sigma}}{d x}}_{T_{4}} \underbrace{+\frac{\overline{S_{d} \Sigma \nabla \cdot \mathbf{n}}}{\bar{u}}}_{T_{5}}
\end{aligned}
$$

and compare terms $T_{4}$ or $T_{5}$, which straightforwardly involve $S_{d}$ and address flamelet collisions and contribution of self-propagation of flamelets to the local stretch rate [72], in cases $\mathrm{H}$ and L.

Figure 8 shows that the magnitude of term $T_{4}$ is low at large $\bar{c}$, with similar results being obtained in cases $\mathrm{H}$ and $\mathrm{L}$. As far as term $T_{5}$ is concerned, it reduces the mean FSD (contributes to a negative $d \bar{\Sigma} / d x$ at large $\bar{c})$ stronger in case $\mathrm{L}$ when compared to $\mathrm{H}$. This difference between the two cases is mainly associated with a significantly higher (lower) mean axial velocity $\bar{u}$ in the denominator of term $T_{5}$ in case $\mathrm{H}(\mathrm{L})$. The observed difference in the behavior of term $T_{4}$ or $T_{5}$ in cases $\mathrm{H}$ and $\mathrm{L}$ at large $\bar{c}$ is not associated with a larger $\langle A\rangle(t)$ in the latter case and, hence, cannot counterbalance the stronger contribution of the DL mechanism to creation of flame surface area at large $\bar{c}$ in case H, see Fig. 4 . 


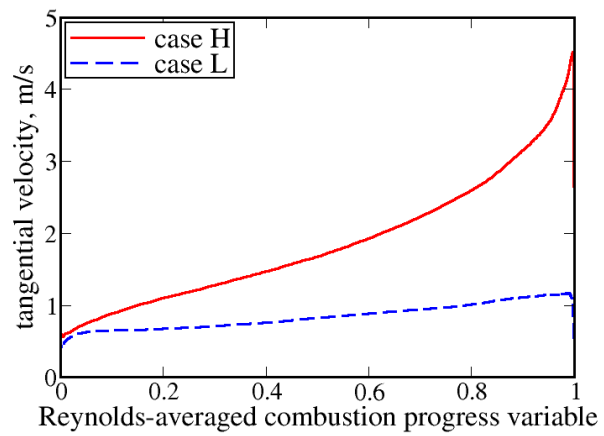

Figure 9: Magnitude $\left\langle\left(\mathbf{v}_{t} \cdot \mathbf{v}_{t}\right)^{1 / 2} \mid 0.01<c<0.05\right\rangle$ of the locally tangential (to flamelet) component $\mathbf{v}_{t}=\mathbf{u}-\mathbf{n}(\mathbf{u} \cdot \mathbf{n})$ of velocity vector $\mathbf{u}$, averaged over the cold edge of flamelets, transverse planes, and time.

\subsection{Locally tangential flow}

Thus, the three previous attempts (Sects. 3.3-3.5) to explain the discussed paradox failed. A clue to solving the problem is seen in Fig. 9, which shows that the magnitude $v_{t}$ of tangential (to flamelet) component $\mathbf{v}_{t}=\mathbf{u}-\mathbf{n}(\mathbf{u} \cdot \mathbf{n})$ of velocity vector $\mathbf{u}$, conditioned to the flamelet leading edge $(0.01<c<$ 0.05), is significantly larger in case $\mathrm{H}$ than in case $\mathrm{L}$. This difference is associated with strong axial acceleration of unburned gas by the mean pressure gradient in case $\mathrm{H}$.

Such a flow acceleration is clearly seen in Fig. 3. A high-speed jet of the unburned gas, indicated in Fig. 3, pushes the local flamelet tip to the right, thus, creating an UMF, which is almost parallel to the $x$-axis in case H, see Fig. 1a. Consequently, at $\bar{c}$ close to unity, i.e. within UMFs, the mean tangential velocity plotted in red solid line in Fig. 9 is close to the local axial velocities $u\left(x, t_{m}\right)$ measured near the finger tip, see points A, B, and $\mathrm{C}$ in Fig. 3. Therefore, $\left\langle v_{t} \mid 0.01<c<0.05\right\rangle$ is large at large $\bar{c}$ in case $\mathrm{H}$.

In case $\mathrm{L},\left\langle v_{t} \mid 0.01<c<0.05\right\rangle$ is significantly lower, because (i) the axial flow acceleration is less pronounced due to a much less magnitude of the conditioned pressure gradient, see Fig. 5 and, moreover, (ii) the finger axis is inclined to the $x$-axis, see Fig. $1 \mathrm{~b}$, thus, making $\left\langle v_{t} \mid 0.01<c<0.05\right\rangle$ lower than $\langle u \mid 0.01<c<0.05\rangle$.

Thus, Figs. 3 and 9 show two effects associated with the axial acceleration of unburned gas due 
to the DL mechanism. On the one hand, near a finger tip, the axial flow velocity of unburned gas is high (see points A, B, and C in Fig. 3) and locally normal to the finger surface, thus, pushing the finger tip to the right and increasing the flamelet surface area. On the other hand, within a finger, the axial flow velocity of unburned gas is also high (see Fig. 3), but it is locally tangential to the side surface of the finger, thus, making $\left\langle v_{t} \mid 0.01<c<0.05\right\rangle$ large. Therefore, the local flow non-uniformities (e.g. small-scale turbulent eddies within a finger or a part of a larger-scale eddy, see the bent shape of a finger in Fig. 1b) could stretch and wrinkle the side surface of the finger during a short residence time, which is required in order for a flow particle to be convected by the local pressure-driven jet from the finger base to the finger tip. Accordingly, such a reduction of the residence time due to the DL mechanism results in mitigating wrinkling of the flamelet surface by turbulent eddies.

In other words, the DL mechanism not only increases flame surface area by inflating an UMF, but also promotes flattening the side surface of the finger by reducing the aforementioned residence time. For instance, using instantaneous axial velocity profiles shown in Fig. 3, one can estimate that the residence time of unburned gas in a finger of a length of $3 \mathrm{~mm}$ in case $\mathrm{H}$ (see Fig. 1a) is on the order of $1 \mathrm{~ms}$ or less, i.e. significantly less than the time scale $\tau_{t}=L / u^{\prime}=7 \mathrm{~ms}$ of turbulence generated in a box and injected through the inlet boundary.

In case $\mathrm{L}$, the residence time is substantially longer, because $\left\langle v_{t} \mid 0.01<c<0.05\right\rangle$ is lower by a factor of about four, whereas a decrease in the finger length is substantially less pronounced, i.e. is about 30 \%, cf. Figs. 1a and 1b (this difference in the lengths of UMFs in cases $\mathrm{H}$ and $\mathrm{L}$ will be discussed in the next subsection). Accordingly, even weaker turbulence experienced by flame L, see Fig. 6, can wrinkle the finger surface by creating additional flame surface area. Such an effect is observed in Fig. 1b where the L-UMF is bent.

Thus, mitigation of turbulent wrinkling of flamelet surface due to reduction of the residence time, caused by the DL mechanism, seems to be relevant to explaining the apparent inconsistency between Figs. 2 and 4 . It is worth noting that mitigation of turbulent wrinkling of a flame surface by a tangential 

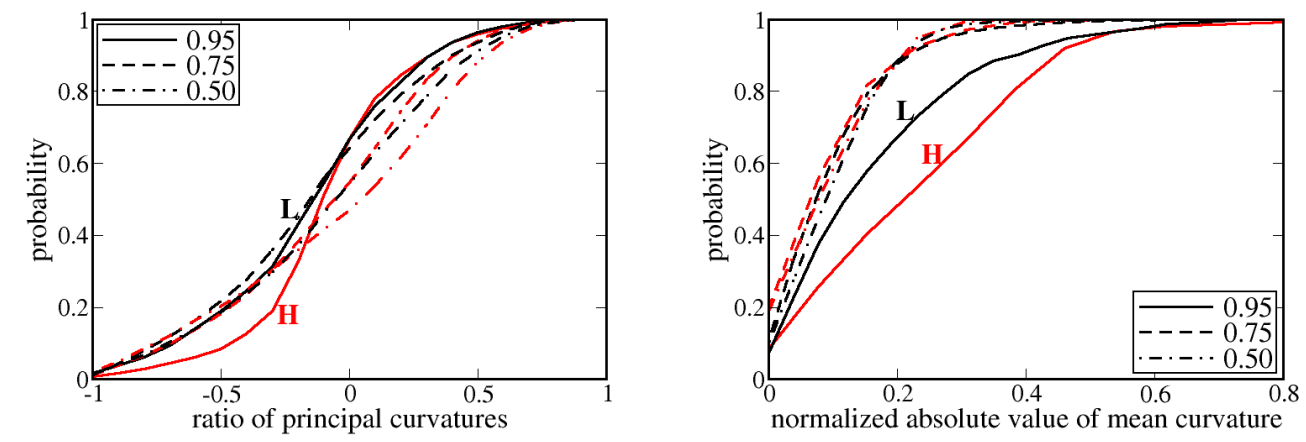

Figure 10: Probabilities (a) of a ratio $\kappa_{1} / \kappa_{2}$ of eigenvalues $\kappa_{1}$ and $\kappa_{2}\left(\left|\kappa_{1}\right| \leq\left|\kappa_{2}\right|\right)$ of the curvature tensor $\partial n_{i} / \partial x_{j}$ and $(\mathrm{b})$ normalized absolute value $\left|\delta_{L} \kappa_{m}\right|$ of the mean curvature $\kappa_{m}=\nabla \cdot \mathbf{n} / 2$, evaluated at three values of the mean combustion progress variable $\bar{c}$ specified in legends. The unit normal vector $\mathbf{n}=-\nabla c /|\nabla c|$ is conditioned to the local combustion progress variable $0.845<c \leq 0.855$ associated with the reaction zone. Results obtained in cases $\mathrm{H}$ and $\mathrm{L}$ are shown in red and black lines, respectively.

stream (or by unsteadiness of the flow in the coordinate framework attached to the stream) was studied $[73,74,75,76,77,78,79]$, but in another context not related to the DL mechanism.

Results of the following two investigations appear to be relevant to further assessing the above hypothesis. First, let us compare curvature statistics in cases H and L both at very large and moderately large values of the mean combustion progress variable $\bar{c}$. The idea of such a study is as follows. If $\bar{c}$ is very large, e.g. $\bar{c}=0.95$, then, reaction zones can reach such a trailing part of the mean flame brush only as elements of UMFs (either their tips or their side surfaces). Accordingly, if the finger surface is smooth, probability of finding negative quasi-cylindrical curvatures should be high. On the contrary, at moderately large values of $\bar{c}$, e.g. $\bar{c}=0.75$ or 0.50 , reaction zones are not associated with UMFs and curvature statistics should be different.

Indeed, Fig. 10a shows that, at $\bar{c}=0.95$ (solid line) in case $\mathrm{H}$, the cumulative probability $\mathcal{P}_{12}=$ $\int_{-1}^{\gamma} P_{12}(\zeta, 0.845 \leq c<0.855, \bar{c}) d \zeta$ is low at $\gamma<-0.35$, but is rapidly increased by $\gamma$ at low absolute values of $|\gamma|$. Here, $P_{12}(\zeta, 0.845 \leq c<0.855, \bar{c})$ is the $\mathrm{PDF}$ for a ratio $\zeta=\kappa_{1} / \kappa_{2}$ of eigenvalues $\kappa_{1}$ and $\kappa_{2}$ (such that $\left|\kappa_{1}\right| \leq\left|\kappa_{2}\right|$ ) of the curvature tensor $\partial n_{i} / \partial x_{j}$, conditioned to the reaction zone, and $\gamma=\kappa_{1} / \kappa_{2}$. Because $\kappa_{1} / \kappa_{2}=0$ in the case of the cylindrical curvature, the discussed curve does indicate that the probability of finding quasi-cylindrical curvatures is high at $\bar{c}=0.95$ in case $\mathrm{H}$. On 
(a)

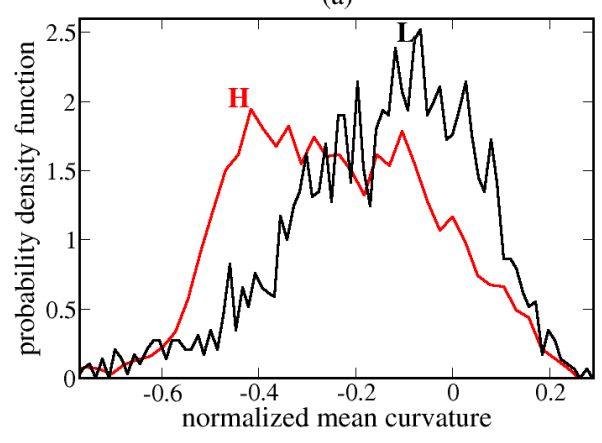

(b)

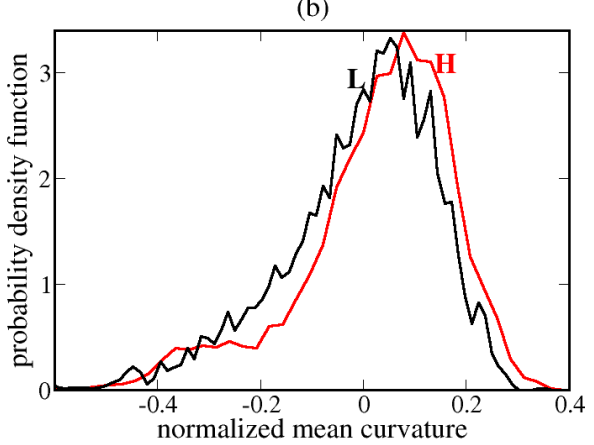

Figure 11: Probability density functions for the normalized mean curvature $\delta_{L} \kappa_{m}$, obtained in cases $\mathrm{H}$ and $\mathrm{L}$ at $(\mathrm{a}) \bar{c}=0.95$ and

(b) $\bar{c}=0.75$. Here, $\kappa_{m}=\nabla \cdot \mathbf{n} / 2$, with the unit normal vector $\mathbf{n}=-\nabla c /|\nabla c|$ being conditioned to the local combustion progress variable $0.845<c \leq 0.855$ associated with the reaction zone.

the contrary, in case $\mathrm{L}$, the slope of the counterpart $\mathcal{P}_{12}(\gamma)$-curve is significantly larger at $\gamma \rightarrow-1$ and does not change much with increasing $\gamma$, thus, indicating that a probability of finding saddle-point curvatures is substantial at $\bar{c}=0.95$ in case $\mathrm{L}$. This difference between the two cases implies that the side surfaces of UMFs are more wrinkled (smooth) in case L $(\mathrm{H})$. As far as moderately large values of $\bar{c}$ are concerned, see dashed and dotted-dashed lines, a qualitative difference between cases $\mathrm{H}$ and $\mathrm{L}$ is hardly visible in Fig. 10a. Moreover, contrary to case H, three curves obtained in case L do not indicate a substantial dependence of $\mathcal{P}_{12}$ on $\bar{c}$ in a range of $0.5 \leq \bar{c} \leq 0.95$.

Figure 10b shows that dependencies of the cumulative probability $\mathcal{P}_{\kappa}=\int_{0}^{\left|\delta_{L} \kappa_{m}\right|} P_{\kappa}(\zeta, 0.845 \leq c<$ $0.855, \bar{c}) d \zeta$ on the normalized absolute value of the mean curvature $\kappa_{m}=\nabla \cdot \mathbf{n} / 2=\left(\kappa_{1}+\kappa_{2}\right) / 2$ look very similar in cases $\mathrm{H}$ and $\mathrm{L}$ both at $\bar{c}=0.50$ and 0.75 , but are substantially different at $\bar{c}=0.95$. This difference in curvature statistics is further shown in Fig. 11a, which indicates that the conditioned $\mathrm{PDF} P_{\kappa}\left(\delta_{L} \kappa_{m}, 0.845 \leq c<0.855, \bar{c}=0.95\right)$ peaks at sufficiently large negative values of $\delta_{L} \kappa_{m}$ in case $\mathrm{H}$ and low values of $\left|\delta_{L} \kappa_{m}\right|$ in case $\mathrm{L}$. This difference between the two cases is also consistent with a hypothesis that the side finger surfaces are more wrinkled (quasi cylindrical) in case L $(\mathrm{H})$. On the contrary, the PDFs $P_{\kappa}\left(\delta_{L} \kappa_{m}, 0.845 \leq c<0.855, \bar{c}=0.75\right)$ look similar in cases H and L, see Fig. 11b, and peak in the vicinity of $\kappa_{m}=0$, in line with numerous earlier DNS data, e.g. see [80] and references 


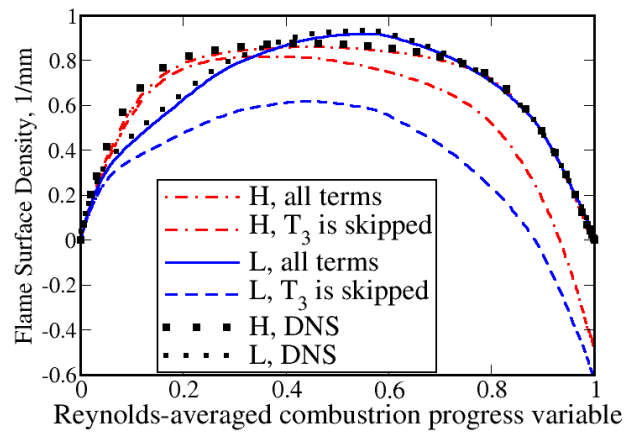

Figure 12: Mean FSD computed in the DNS (symbols) and evaluated by integrating Eq. (8) either by extracting all terms on the RHS from the DNS (dotted-dashed and solid lines) or by extracting all terms on the RHS from the DNS, but skipping term $T_{3}$ (double-dashed-dotted and dashed lines).

therein.

Second, let us compare a role played by term $T_{3}$ in Eq. (8) in cases $\mathrm{H}$ and $\mathrm{L}$, because this term is associated with an increase in FSD by turbulent straining. For this purpose, we computed the following quantities. First, we extracted the mean FSD $\bar{\Sigma}=\overline{|\nabla c|}$ straightforwardly from the DNS data. Results obtained in cases $\mathrm{H}$ and $\mathrm{L}$, are shown in larger and smaller symbols, respectively, in Fig. 12. These dependencies of $\bar{\Sigma}$ on $\bar{c}$ are basically similar in the two cases, in line with the fact that both $\langle A\rangle(t)$ and $\left\langle\delta_{t, 1}\right\rangle(t)$ oscillate in comparable ranges in cases $\mathrm{H}$ and $\mathrm{L}$, see Fig. 2.

Second, we computed $\bar{\Sigma}(\bar{c})$ by numerically integrating Eq. (8), with all terms on the Right Hand Side (RHS) being extracted from the DNS data. Results shown in red dotted-dashed and blue solid lines in cases $\mathrm{H}$ and $\mathrm{L}$, respectively, agree very well with $\bar{\Sigma}(\bar{c})$ extracted straightforwardly from the same DNS data (symbols), thus, validating the integration method.

Third, we repeated the numerical integration of Eq. (8), but skipped term $T_{3}$ on the RHS. Results shown in red double-dashed-dotted and blue dashed lines ${ }^{4}$ in cases $\mathrm{H}$ and $\mathrm{L}$, respectively, indicate that the neglect of $T_{3}$ changes the FSD stronger in case $\mathrm{L}$ when compared to $\mathrm{H}$. Therefore, the strain term $T_{3}$

\footnotetext{
${ }^{4}$ These lines do not go to zero at $\bar{c} \rightarrow 1$, because they show results of integration of a wrong transport equation (term $T_{3}$ is skipped).
} 
plays a more important role in flame $\mathrm{L}$, in line with the above hypothesis about mitigation of turbulent wrinkling of flamelet surface by the DL mechanism in case $\mathrm{H}$.

At first glance, this result might appear to be inconsistent with Figs. 6 and 7, which indicate that the reaction zone is subject to more intense turbulence in case $\mathrm{H}$ when compared to case L. However, it is worth remembering that (i) the derivative $d \bar{\Sigma} / d x$ is controlled by term $\overline{a_{t} \Sigma}$ divided by the mean axial velocity $\bar{u}$, see Eq. 8, and, (ii) due to a very large difference in the mean axial pressure gradients, see Fig. 5, this velocity is significantly higher in case $\mathrm{H}$, thus, making the magnitude of $T_{3}$ lower. This explanation again highlights the difference between axial flow accelerations in cases $\mathrm{H}$ and $\mathrm{L}$ and, thus, is in line with our earlier arguments.

While, for clarity, the focus of the above discussion was placed on turbulence within an UMF, a stronger axial acceleration of the unburned flow can also impede creating flame surface area in other zones of the mean flame brush. For any finite value $c^{*}$, a travel time $t^{*}$, which is required for a turbulent eddy to move from the leading edge of the mean flame brush to a plane of $x=x^{*}$ such that $\bar{c}\left(x^{*}\right)=c^{*}$, is shorter if the axial flow acceleration is stronger. Accordingly, one effect of an increase in the density ratio consists of decreasing $\bar{\Sigma}\left(c^{*}\right)$ due to a decrease of time $t^{*}$ during that turbulent eddies can wrinkle the flame front at $\bar{c}<c^{*}$.

Such an effect is clearly seen on the RHS of Eq. (8), where each term is divided by $\bar{u}$, which is increased by $\sigma$. Therefore, substitution of $T_{3}=0$ into Eq. (8) reduces $\bar{\Sigma}$ stronger in case L when compared to case $\mathrm{H}$ not only at large $\bar{c}$, where the unburned gas may only appear within an UMF, but also in the entire flame brush, cf. curves shown in red double-dashed-dotted and blue dashed lines in Fig. 12. In other words, contribution of turbulent straining term $\overline{a_{t} \Sigma} / \bar{u}$ to production of flame surface area is stronger (weaker) in case $\mathrm{L}(\mathrm{H})$ even in the middle of the flame brush.

Comparison of curves plotted in red double-dashed-dotted and blue dashed lines in Fig. 12 shows also that the difference between the two curves is reduced when $\bar{c} \rightarrow 1$, i.e., at $\bar{c} \approx 1$, term $T_{3}$ plays a more important role in case $\mathrm{H}$. This trend is associated with high strain rates created near finger tips 
in case H. Indeed, Fig. 7 indicates that, at $\bar{c}=0.9,\left\langle a_{t} \mid c=0.85\right\rangle$ is larger by a factor of about five in case $\mathrm{H}$ when compared to case $\mathrm{L}$ (note that $\tau_{c}$ is larger by a factor of two in case $\mathrm{L}$ ). Therefore, the difference in the local $\left\langle a_{t} \mid c=0.85\right\rangle$ at $\bar{c} \approx 1$ is larger than the difference in the unburned gas velocities $u_{u}$ upstream of a finger tip. Consequently, the magnitude of the residence-time effect is insufficient in order to overwhelm the large difference in the local strain rates at $\bar{c} \approx 1$.

\subsection{Finger evolution}

In the above discussion, the mitigation effect is attributed to reduction of the residence time of unburned turbulent flow within the mean flame brush (e.g. within an UMF) due to the flow acceleration by the axial pressure gradient. Accordingly, there is a question why the residence-time reduction due to more pronounced axial flow acceleration in the case of a larger $\sigma$ is not counterbalanced by an increase in the UMF length. Indeed, as shown in Figs. 1a and 1b, the computed finger lengths are comparable (30\% difference) in cases $\mathrm{H}$ and $\mathrm{L}$ in spite of the fact that the $\mathrm{H}-\mathrm{UMF}$ is inflated by a much stronger jet of unburned gas.

To answer the question, the normalized local displacement speed $\rho S_{d} /\left(\rho_{u} S_{L}\right)$ and the normalized magnitude $u_{n}=\left|\rho \mathbf{u} \cdot \mathbf{n} /\left(\rho_{u} S_{L}\right)\right|$ of the locally normal gas velocity were computed at finger tips at various instants. Typical results are reported in Fig. 13, which shows different phases of finger-tip evolution. It is worth remembering that the coordinates $\mathbf{x}_{f t}$ of the finger tip are associated with the reaction zone and, therefore, a low density. Accordingly, the velocity $\rho_{u} u_{n} / \rho$ is higher than $u_{u}$ evaluated at instants A, B, or C (see Fig. 3) in spite of the fact that the latter velocity is also locally normal to the same flamelet element.

During the first (finger growth) relatively long phase, $u_{n}>S_{d}$, cf. circles and triangles at $t^{\prime}<-0.4$ ms, and the finger is inflated by the unburned gas. During this phase, the local flamelet structure is weakly perturbed, e.g. see iso-contour of $c\left(x, y_{f t}, z, t\right)=0.1$, shown in dashed line in Fig. 1a at instant $t=18.60 \mathrm{~ms}$, which is labeled with A in Fig. 13a. Moreover, crosses in Fig. 13a indicate that the axial distance between the $\mathrm{H}$-finger tip and the nearest point characterized by $c\left(x, y_{f t}, z_{f t}, t\right) \leq 0.1$ remains 
(a) case $\mathrm{H}$

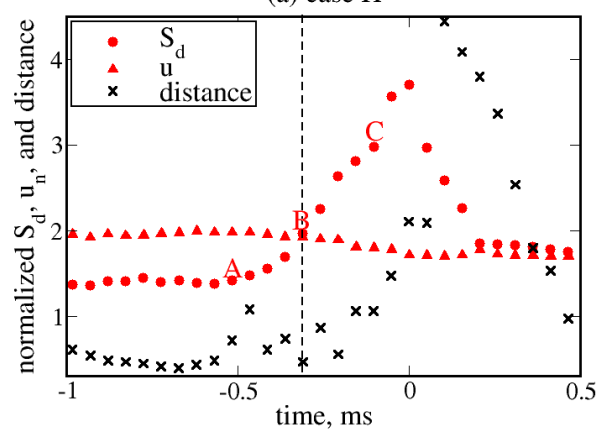

(b) case L

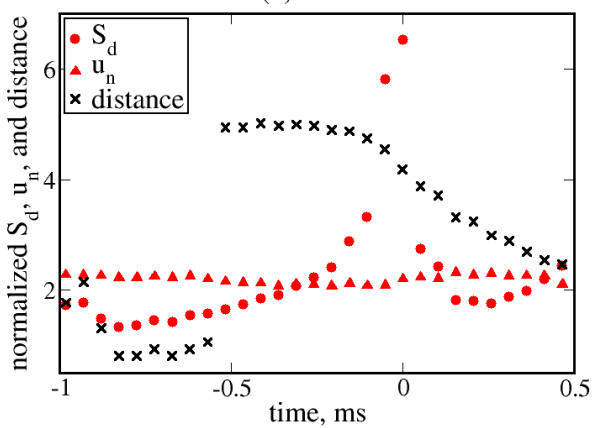

Figure 13: Normalized local displacement speed $\rho S_{d} /\left(\rho_{u} S_{L}\right)$ (circles) and magnitude $u_{n}=\left|\rho \mathbf{u} \cdot \mathbf{n} /\left(\rho_{u} S_{L}\right)\right|$ of the local normal velocity (triangles), both evaluated at finger tip, as well as normalized axial distance $\Delta x / \delta_{L}$ (crosses) between the finger tip and the closest point characterized by $c\left(x, y_{f t}, z_{f t}, t\right) \leq 0.1$. The distance is divided by a factor of four. Time is shifted, i.e. $t^{\prime}=t-\Delta t$, in order for $S_{d}$ to peak at $t^{\prime}=0$. Letters A, B, and C corresponds to instants A, B, and C, respectively, in Fig. 3 .

low.

At the end of this phase $\left(t^{\prime} \approx-0.5 \mathrm{~ms}\right)$, the local displacement speed (circles) starts increasing so that $S_{d}=u_{n}$ at instant B in Fig. 13a. This increase in $S_{d}\left(t^{\prime}\right)$ is controlled by two mechanisms. First, the growth of the finger length is accompanied by a decrease in the transverse width of the finger and an increase in the magnitude of the curvature of its tip, see crosses in Fig. 14 or cf. two $W_{c}$-iso-lines shown in Fig. 1a in case $\mathrm{H}$ (these iso-lines are associated with instants A and B, respectively, in Fig. 13). The increase in the curvature magnitude $\left(\nabla \cdot \mathbf{n}\right.$ is negative at a finger tip) promotes increasing $S_{d}$.

Second, gas within the finger is preheated similarly to the well-known preheating of unburned mixture near the tip of a laminar Bunsen flame [81] or near cusps in premixed turbulent flames [67]. For instance, crosses in Fig. 13a show that axial distance between the finger tip and the closest point characterized by $c \leq 0.1$ is large at $-0.3<t^{\prime}<0.5$ and, in particular, is significantly larger than the counterpart distance in the unperturbed laminar flame, thus, indicating substantial thickening of the preheat zone. The same thickening effect can be seen by comparing dotted-dashed lines $(t=18.8 \mathrm{~ms})$ in Fig. 1a.

Mechanisms that control the rapid growth of the local $S_{d}$ at the finger tip are addressed in Fig. 14, which shows the normalized curvature $\delta_{L} \nabla \cdot \mathbf{n}$ (crosses) and various terms in the well-known decompo- 
(a) case $\mathrm{H}$

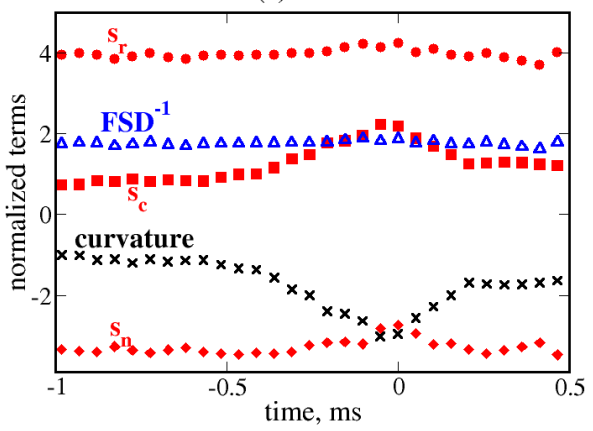

(b) case L

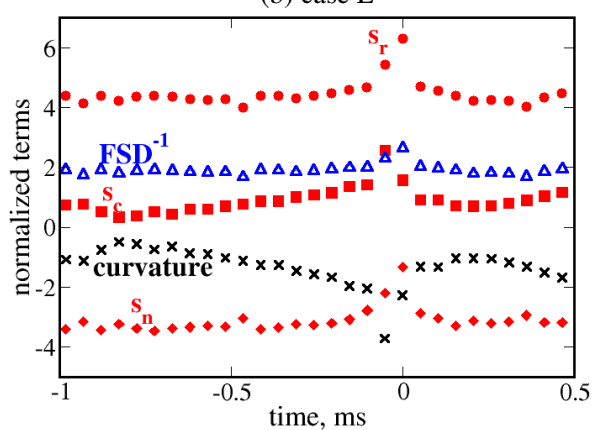

Figure 14: Evolution of various quantities evaluated at finger tip, with time being shifted, i.e. $t^{\prime}=t-\Delta t$, in order for $S_{d}$ to peak at $t^{\prime}=0$. Filled symbols show contributions to $\rho S_{d} /\left(\rho_{u} S_{L}\right)$ from reaction (circles), curvature (squares), and normal diffusion (diamonds) terms. Triangles show $1 /\left(\delta_{L}|\nabla c|\right)$. Crosses show $\delta_{L} \nabla \cdot \mathbf{n}$.

sition [82] of the local displacement speed

$$
S_{d}=\frac{\nabla \cdot(\rho D \nabla c)+W_{c}}{\rho|\nabla c|}=\underbrace{-D \nabla \cdot \mathbf{n}}_{s_{c}}+\underbrace{\frac{\mathbf{n} \cdot \nabla(\rho D \mathbf{n} \cdot \nabla c)}{\rho|\nabla c|}}_{s_{n}}+\underbrace{\frac{W_{c}}{\rho|\nabla c|}}_{s_{r}} .
$$

Open triangles indicate that the term $1 /\left(\delta_{L}|\nabla c|\right)$ varies weakly during finger evolution. An increase in the magnitude of the negative local curvature (crosses) around $t^{\prime}=0$ is accompanied by an increase in the magnitude of the positive curvature term $s_{c}$ (squares) and a decrease in the magnitude of the negative normal diffusion term $s_{n}$ (diamonds). These two effects control the rapid increase in $S_{d}$ around $t^{\prime}=0$, see circles in Fig. 13, but an increase in $1 /\left(\delta_{L}|\nabla c|\right)$ contributes also to an increase in the reaction term $s_{r}$ (circles) in case L at $t^{\prime}=0$, see Fig. $14 \mathrm{~b}$. The facts that (i) the term $1 /\left(\delta_{L}|\nabla c|\right)$ varies weakly, (ii) an increase in $S_{d}$ starts substantially earlier than an increase in the distance $\Delta x$ in case $\mathrm{H}$, cf. circles and crosses in Fig. 13a, and (iii) an increase in $S_{d}$ starts significantly later than an increase in the distance $\Delta x$ in case L, cf. circles and crosses in Fig. 13b, imply that the increase in $S_{d}$ around $t^{\prime}=0$ is mainly controlled by the curvature effect, whereas contribution of preheating to the increase in the local displacement speed of the finger tip is of minor importance under conditions of the present study.

Due to the discussed effects, the local displacement speed exceeds the normal flow velocity, e.g. at instant B in Fig. 13a, and the finger tip starts propagating towards the leading edge of the mean flame 
brush, with the difference in $S_{d}$ and $u_{n}$ being large, cf. circles and triangles in Fig. 13 at $t^{\prime}=0$. Due to the large difference in $S_{d}$ and $u_{n}$, the finger-disappearance phase is relatively short, followed by a drop in $S_{d}$ when the finger vanishes. Comparison of Figs. 13a and 13b or Figs. 14a and 14b shows that $S_{d}$ or $u_{n}$ behaves similarly in case $\mathrm{H}$ and $\mathrm{L}$.

The discussed results imply that an increase in $S_{d}$ at the strongly-curved tip of a thin finger occurs so fast that even a high flow velocity is rapidly overwhelmed.

Moreover, because the local displacement speed is inversely proportional to the density, $S_{d}$ increases significantly from the leading edge of a flamelet to its reaction zone in case H. On the contrary, an increase in the local normal velocity with $c$ is much less pronounced near a strongly curved finger tip when compared to the unperturbed laminar flame. Indeed, $u_{u}$ in point $\mathrm{C}$ in Fig. 3 is locally normal to the finger tip and is less than the local velocity $|\mathbf{u} \cdot \mathbf{n}|=\rho_{u} u_{n} / \rho$ reported in Fig. 13 by a factor of about 1.7, whereas the difference in the counterpart velocities obtained from the unperturbed planar laminar flame is much larger in case $\mathrm{H}(\sigma=7.53)$. In case $\mathrm{L}$, such a difference between the values of $S_{d}+\mathbf{u} \cdot \mathbf{n}$, evaluated in unburned gas near a finger tip and in the reaction zone at the finger tip, is substantially less due to a low density ratio $(\sigma=2.5)$. Accordingly, in spite of the fact that the speed of an unburned-gas jet within an UMF is significantly higher in case $\mathrm{H}$ when compared to L, the discussed difference between the $c$-dependencies of $S_{d}$ and $|\mathbf{u} \cdot \mathbf{n}|$ in the vicinity of the finger tip contributes also to the ability of the finger tip to propagate against the high-speed jet not only in case L, but also in case $\mathrm{H}$.

\subsection{A comment on the net effect of the DL mechanism}

The above analysis of DNS data indicates that, under weakly turbulent conditions, the DL mechanism not only straightforwardly increases burning rate by inflating UMFs and creating flame surface area, but also indirectly reduces the burning rate by mitigating creation of flame surface area by turbulent eddies. To the best of the present authors' knowledge, such a reduction effect of the DL mechanism on turbulent burning rate has not yet been discussed in the literature. To the contrary, models that 
deal with the DL instability of thin inherently laminar flamelets in a turbulent flow are explicitly or implicitly based on an assumption that "external turbulence and the DL instability work together" [83], although Akkerman and Bychkov [83] and Bychkov et al. [84] noted that response of a premixed flame to "vortices perpendicular to the direction of flame propagation" could be more pronounced at low density ratios.

It is worth stressing, however, that the discussed weakly turbulent DNS data should not be considered to show that the net effect of the DL mechanism on premixed turbulent flame speed is of minor importance. First, even if the two manifestations of this mechanism, i.e. UMFs and the aforementioned mitigation effect, almost completely counterbalance one another as far as the net flame surface area is concerned, the two studied particular cases (H and L) are obviously not sufficient in order to expect that the two manifestations of the DL mechanism counterbalance one another in a general case. Further research into the issue is definitely required, e.g. by analyzing DNS data obtained in a wide range of substantially different conditions.

Second, strictly speaking, even in the two considered particular cases, the obtained results do not show that the net influence of the DL mechanism on $U_{t} / S_{L}$ is weak. To draw such a conclusion (or reject it), DNS of the constant-density case should be run. The present results solely indicate that the net influence of the DL mechanism on $U_{t} / S_{L}$ is almost the same at two significantly different density ratios, i.e. $\bar{U}_{t} / S_{L}=1.88$ and 1.77 at $\sigma=7.53$ and 2.5 , respectively. However, Figs. 1,2 , and 4 clearly show that the DL mechanism well manifests itself even at $\sigma=2.5$, i.e. in this sense, the present results are consistent with earlier DNS studies $[21,22,23,24,25,26,27]$ that indicated an importance of the DL mechanism at $u^{\prime} / S_{L}=O(1)$.

As far as the weak influence of $\sigma$ on the fully-developed turbulent burning velocity $\bar{U}_{t}$ is concerned, this issue was not addressed in Refs. [21, 22, 26]. Table 1 and Fig. 10 reported by Treurniet et al. [23] imply such an influence, but their flames characterized by $\sigma=2,4$, and 6 experienced different $u^{\prime}$ at each $\sigma$. Moreover, in the cited study, flame propagation was modeled by solving a level set equation 
and invoking a constant (stretch-independent) $S_{L}$. Under the latter simplification, the flame sheet is unstable with respect to the entire spectrum of perturbations, with the highest growth rate of the DL instability being obtained for perturbations with the shortest wavelength $[12,13]$. On the contrary, a laminar flame with a finite thickness is well known to be stable with respect to perturbations whose wavelength is less than a neutral wavelength $\lambda_{c r}[25,26,27]$, with $\lambda_{c r}$ being as large as $70 D_{u} / S_{L}[85]$. Accordingly, the highest possible growth rate of the DL instability associated with the present DNS appears to be much less than the counterpart growth rate associated with the simulations by Treurniet et al. [23]. Therefore, the latter DNSs are likely to overestimate the influence of the DL instability on $\bar{U}_{t}$.

Matalon et al. [24, 25, 26, 27] performed a 2D DNS study of weakly turbulent flames by solving a level set equation, with dependence of the flame speed on the local stretch rate being taken into account. These authors reported several figures that showed an influence of $\sigma$ on $\bar{U}_{t}$. However, Fig. 9 in [24] and Figs. 3-5 in [25] are relevant to "subcritical" conditions, i.e. $\lambda_{c r}$ is larger than the width of the computational domain and, hence, the DL instability cannot develop. Under "supercritical" conditions, the DL instability can develop and $\bar{U}_{t}$ computed by Fogla et al. [27] is increased by $\sigma$, see Figs. 10 and 11 in the cited paper. However, the effect is weak, i.e. difference in $\bar{U}_{t} / S_{L}$ obtained at $\sigma=2$ and 6 is about $10 \%$. This difference is comparable with the $5 \%$ difference simulated in the present work, i.e. $\bar{U}_{t} / S_{L}=1.88$ and 1.77 at $\sigma=7.53$ and 2.5 , respectively. Therefore, the present DNS data are consistent with the DNS data by Matalon et al. [24, 25, 26, 27].

To conclude this comparison with earlier studies, we may also remind that neither approximations of the most extensive experimental databases $[1,2,3,4,5]$ nor a few target-directed experimental studies $[6,7]$ indicate a substantial effect of $\sigma$ on turbulent flame speed. 


\section{Conclusion}

The present DNS study evidences that acceleration of unburned gas flow due to combustion-induced pressure gradient not only increases flame surface area by creating unburned mixture fingers and pushing the finger tip into unburned gas, but also mitigates turbulent wrinkling of the flame surface (e.g. flattens the side surface of the finger) by reducing residence time during that turbulent eddies can affect the surface (in particular, the side surface of the finger).

\section{Acknowledgments}

AL gratefully acknowledges the financial support by the Combustion Engine Research Center and Chalmers Transport and Energy Areas of Advance. VS gratefully acknowledges the financial support by ONERA.

\section{References}

[1] R.G. Abdel-Gayed, D.Bradley, M. Lawes, Turbulent burning velocities: a general correlation in terms of straining rates, Proc. R. Soc. London A 414 (1987) 389-413.

[2] Ö.L. Gülder, Turbulent premixed flame propagation models for different combustion regimes, Symp. (Int.) Combust. 23 (1990) 743-750.

[3] D. Bradley, A.K.C. Lau, M. Lawes, Flame stretch rate as a determinant of turbulent burning velocity, Phil. Trans. R. Soc. London A 338 (1992) 359-387.

[4] A.N. Lipatnikov, J. Chomiak, Turbulent flame speed and thickness: phenomenology, evaluation, and application in multi-dimensional simulations, Prog. Energy Combust. Sci. 28 (2002) 1-74.

[5] F. Wu, A. Saha, S. Chaudhuri, C.K. Law, Propagation speeds of expanding turbulent flames of C4 to C8 n-alkanes at elevated pressures: experimental determination, fuel similarity, and stretch-affected local extinction, Proc. Combust. Inst. 35 (2015) 1501-1508.

[6] A.A. Burluka, J.F. Griffiths, K. Liu, M. Orms, Experimental studies of the role of chemical kinetics in turbulent flames, Combust. Explos. Shock Waves 45 (2009) 383-391.

[7] A.N. Lipatnikov, W.Y. Li, L.J. Jiang, S.S. Shy, Does density ratio significantly affect turbulent flame speed? Flow Turbulence Combust. 2016, submitted.

[8] B. Karlovitz, D.W. Denniston, F.E. Wells, Investigation of turbulent flames, J. Chem. Phys. 19 (1951) 541-547.

[9] A.C. Scurlock, J.H. Grover, Propagation of turbulent flames, Symp. (Int.) Combust. 4 (1953) 645658.

[10] A.N. Lipatnikov, J. Chomiak, Effects of premixed flames on turbulence and turbulent scalar transport, Prog. Energy Combust. Sci. 36 (2010) 1-102. 
[11] V.A. Sabelnikov, A.N. Lipatnikov, Recent advances in understanding of thermal expansion effects in premixed turbulent flames, Annu. Rev. Fluid Mech. 49 (2017) 91-117.

[12] G. Darrieus, Propagation d'un front de flamme, Unpublished work presented in 1938 at La Technique Moderne (Paris) and in 1945 at Congrés de Mećanique Appliqueé (Paris).

[13] L.D. Landau, On the theory of slow combustion, Acta Psysicochimica USSR 19 (1944) 77-85.

[14] V.R. Kuznetsov, Effect of flame instability on turbulent combustion of a homogeneous mixture, In: Combustion of gases and natural fuels. Chernogolovka: OIKhF, 1980, pp.32-37 (in Russian).

[15] V.R. Kuznetsov, Limiting laws of propagation of a turbulent flame, Combust. Explos. Shock Waves 18 (1982) 172-179.

[16] R.N. Paul, K.N.C. Bray, Study of premixed turbulent combustion including Landau-Darrieus instability effects, Symp. (Int.) Combust. 26 (1996) 259-266.

[17] A.Y. Klimenko, Examining the cascade hypothesis for turbulent premixed combustion, Combust. Sci. and Technol. 139 (1998) 15-40.

[18] V. Bychkov, Importance of the Darrieus-Landau instability for strongly corrugated turbulent flames, Phys. Rev. E 68 (2003) 066304.

[19] V. Akkerman, V. Bychkov, Turbulent flame and the Darrieus-Landau instability in a threedimensional flow, Combust. Theory Modelling 7 (2003) 767-794.

[20] S. Chaudhuri, V. Akkerman, C.K. Law, Spectral formulation of turbulent flame speed with consideration of hydrodynamic instability, Phys. Rev. E 84 (2011) 026322.

[21] H. Boughanem, A. Trouvé, The domain of influence of flame instabilities in turbulent premixed combustion, Proc. Combust. Inst. 27 (1998) 971-978.

[22] N. Peters, H. Wenzel, F.A. Williams, Modification of the turbulent burning velocity by gas expansion, Proc. Combust. Inst. 28 (2000) 235-243.

[23] T.C. Treurniet, F.T.M. Nieuwstadt, B.J. Boersma, Direct numerical simulation of homogeneous turbulence in combination with premixed combustion at low Mach number modelled by the Gequation, J. Fluid Mech. 565 (2006) 25-62.

[24] F. Creta, M. Matalon, Propagation of wrinkled turbulent flames in the context of hydrodynamic theory, J. Fluid Mech. 680 (2011) 225-264.

[25] N. Fogla, F. Creta, M. Matalon, Influence of the Darrieus-Landau instability on the propagation of planar turbulent flames, Proc. Combust. Inst. 34 (2013) 1509-1517.

[26] N. Fogla, F. Creta, M. Matalon, Effect of folds and pockets on the topology and propagation of premixed turbulent flames, Combust. Flame 162 (2015) 2758-2777.

[27] N. Fogla, F. Creta, M. Matalon, The turbulent flame speed for low-to-moderate turbulence intensities: Hydrodynamic theory vs. experiments. Combust. Flame 2016, in press.

[28] H. Kobayashi, T. Tamura, K. Maruta, T. Niioka, F.A. Williams, Burning velocity of turbulent premixed flames in a high-pressure environment, Symp. (Int.) Combust. 26 (1996) 389-396.

[29] H. Kobayashi, H. Kawazoe, Flame instability effects on the smallest wrinkling scale and burning velocity of high-pressure turbulent premixed flames, Proc. Combust. Inst. 28 (2000) 375-382.

[30] A.M. Steinberg, J.F. Driscoll, Straining and wrinkling processes during turbulence-premixed flame interaction measured using temporally-resolved diagnostics, Combust. Flame 156 (2009) 2285-2306.

[31] G. Troiani, F. Creta, M. Matalon, Experimental investigation of Darrieus-Landau instability effects on turbulent premixed flames, Proc. Combust. Inst. 35 (2015) 1451-1459. 
[32] A.N. Lipatnikov, J. Chomiak, Molecular transport effects on turbulent flame propagation and structure, Prog. Energy Combust. Sci. 31 (2005) 1-73.

[33] V.R. Kuznetsov, V.A. Sabelnikov, Turbulence and combustion, Hemisphere, New York, 1990.

[34] J. Chomiak, Combustion: a study in Theory, Fact and Application, Gordon \& Breach, New York, 1990.

[35] A. Lipatnikov, Fundamentals of premixed turbulent combustion, CRC Press, Boca Raton, Florida, 2012.

[36] S. Nishiki, T. Hasegawa, R. Borghi, R. Himeno, Modeling of flame-generated turbulence based on direct numerical simulation databases, Proc. Combust. Inst. 29 (2002) 2017-2022.

[37] S. Nishiki, T. Hasegawa, R. Borghi, R. Himeno, Modelling of turbulent scalar flux in turbulent premixed flames based on DNS databases, Combust. Theory Modelling 10 (2006) 39-55.

[38] Y.H. Im, K.Y. Huh, S. Nishiki, T. Hasegawa, Zone conditional assessment of flame-generated turbulence with DNS database of a turbulent premixed flame, Combust. Flame 137 (2004) 478-488.

[39] A. Mura, K. Tsuboi, T. Hasegawa, Modelling of the correlation between velocity and reactive scalar gradients in turbulent premixed flames based on DNS data, Combust. Theory Modelling 12 (2008) 671-698.

[40] A. Mura, V. Robin, M. Champion, T. Hasegawa, Small scale features of velocity and scalar fields in turbulent premixed flames, Flow Turbul. Combust. 82 (2009) 339-358.

[41] V. Robin, A. Mura, M. Champion, T. Hasegawa, Modeling of the effects of thermal expansion on scalar turbulent fluxes in turbulent premixed flames, Combust. Sci. Technol. 182 (2010) 449-464.

[42] V. Robin, A. Mura, M. Champion, Direct and indirect thermal expansion effects in turbulent premixed flames, J. Fluid Mech. 689 (2011) 149-182.

[43] K.N.C. Bray, M. Champion, P.A. Libby, N. Swaminathan, Scalar dissipation and mean reaction rates in premixed turbulent combustion, Combust. Flame 158 (2011) 2017-2022.

[44] A.N. Lipatnikov, S. Nishiki, T. Hasegawa, A direct numerical simulation study of vorticity transformation in weakly turbulent premixed flames, Phys. Fluids 26 (2014) 105104.

[45] A.N. Lipatnikov, V.A. Sabelnikov, S. Nishiki, T. Hasegawa, N. Chakraborty, DNS assessment of a simple model for evaluating velocity conditioned to unburned gas in premixed turbulent flames, Flow Turbul. Combust. 94 (2015) 513-526.

[46] A.N. Lipatnikov, S. Nishiki, T. Hasegawa, DNS assessment of relation between mean reaction and scalar dissipation rates in the flamelet regime of premixed turbulent combustion, Combust. Theory Modelling 19 (2015) 309-328.

[47] A.N. Lipatnikov, J. Chomiak, V.A. Sabelnikov, S. Nishiki, T. Hasegawa, Unburned mixture fingers in premixed turbulent flames, Proc. Combust. Inst. 35 (2015) 1401-1408.

[48] V.A. Sabelnikov, A.N. Lipatnikov, N. Chakraborty, S. Nishiki, T Hasegawa, A transport equation for reaction rate in turbulent flows, Phys. Fluids 28 (2016) 081701.

[49] A.N. Lipatnikov, V.A. Sabelnikov, S. Nishiki, T. Hasegawa, Flamelet perturbations and flame surface density transport in weakly turbulent premixed combustion, Combust. Theory Modelling, 2016 , in press.

[50] V.A. Sabelnikov, A.N. Lipatnikov, N. Chakraborty, S. Nishiki, T Hasegawa, A balance equation for the mean rate of product creation in premixed turbulent flames, Proc. Combust. Inst. 36, in press.

[51] A.Y. Poludnenko, Pulsating instability and self-acceleration of fast turbulent flames, Phys. Fluids 27 (2015) 014106. 
[52] A. Buschmann, F. Dinkelacker, T. Schäfer, M. Schäfer, J. Wolfrum, Measurement of the instantaneous detailed flame structure in turbulent premixed combustion, Symp. (Int.) on Combust. 26 (1996) 437-445.

[53] I.G. Shepherd, R.K. Cheng, The burning rate of premixed flames in moderate and intense turbulence, Combust. Flame 127 (2001) 2066-2075.

[54] R.K. Cheng, I.G. Shepherd, B. Bédat, L. Talbot, Premixed turbulent flame structures in moderate and intense isotropic turbulence, Combust. Sci. Technol. 174 (2002) 29-59.

[55] Y.-C. Chen, R.W. Bilger, Experimental investigation of three-dimensional flame-front structure in premixed turbulent combustion - I: Hydrocarbon/air Bunsen flames, Combust. Flame 131 (2002) 400-435.

[56] S.A. Filatyev, M.P. Thariyan, R.P. Lucht, J.P. Gore, Simultaneous stereo particle image velocimetry and double-pulsed planar laser-induced fluorescence of turbulent premixed flames, Combust. Flame 150 (2007) 201-209.

[57] A.M. Steinberg, J.F. Driscoll, S.L. Ceccio, Measurements of turbulent premixed flame dynamics using cinema stereoscopic PIV, Exp. Fluids 44 (2008) 985-999.

[58] G. Hartung, J. Hult, R. Balachandran, N.R. Mackley, C.F. Kaminski, Flame front tracking in turbulent lean premixed flames using stereo PIV and time-sequenced planar LIF of OH, Appl. Physics B 96 (2009) 843-862.

[59] S. Kheirkhah, Ö.L. Gülder, Turbulent premixed combustion in V-shaped flames: characteristics of flame front, Phys. Fluids 25 (2013) 055107.

[60] J. Wang, M. Zhang, Z. Huang, T. Kudo, H. Kobayashi, Measurement of the instantaneous flame front structure of syngas turbulent premixed flames at high pressure, Combust. Flame 160 (2013) $2434-2441$.

[61] S. Daniele, J. Mantzaras, P. Jansohn, A. Denisov, K. Boulouchos, Flame front/turbulence interaction for syngas fuels in the thin reaction zones regime: turbulent and stretched laminar flame speeds at elevated pressures and temperatures, J. Fluid Mech. 724 (2013) 36-68.

[62] M. Day, S. Tachibana, J. Bell, M. Lijewski, V. Beckner, R.K. Cheng, A combined computational and experimental characterization of lean premixed low swirl laboratory flames: II. Hydrogen flames, Combust. Flame 162 (2015) 2148-2165.

[63] M. Zhang, J. Wang, W. Jin, Z. Huang, H. Kobayashi, L. Ma, Estimation of 3D flame surface density and global fuel consumption rate from 2D PLIF images of turbulent premixed flame, Combust. Flame 162 (2015) 2087-2097.

[64] C.D. Carter, S. Hammack, T. Lee, High-speed flame-front imaging in premixed turbulent flames using laser-induced fluorescence of the CH C-X band, Combust. Flame 168 (2016) 66-74.

[65] J.B. Bell, M.S. Day, J.F. Grcar, M.J. Lijewski, Active control for statistically stationary turbulent premixed flame simulations, Comm. Appl. Math. Comput. Sci. 1 (2006) 29-51.

[66] A.Y. Poludnenko, E.S. Oran, The interaction of high-speed turbulence with flames: global properties and internal flame structure, Combust. Flame 157 (2010) 995-1011.

[67] A.Y. Poludnenko, E.S. Oran, The interaction of high-speed turbulence with flames: turbulent flame speed, Combust. Flame 158 (2011) 301-326.

[68] P.E. Hamlington, A.Y. Poludnenko, E.S. Oran, Interactions between turbulence and flames in premixed reacting flows, Phys. Fluids 23 (2011) 125111.

[69] A.J. Aspden, M. Day, J. Bell, Turbulence-flame interactions in lean premixed hydrogen: transition to the distributed burning regime, J. Fluid. Mech. 680 (2011) 287-320. 
[70] J. Kwon, K.Y. Huh, Effects of Lewis number, density ratio and gravity on burning velocity and conditional statistics in stagnating turbulent premixed flames, Combust. Theory Modelling 18 (2014) $501-514$.

[71] S. Lapointe, B. Savard, G. Blanquart, Differential diffusion effects, distributed burning, and local extinction in high Karlovitz premixed flames, Combust. Flame 162 (2015) 3341-3355.

[72] D. Veynante, L. Vervisch, Turbulent combustion modeling, Prog. Energy Combust. Sci. 28 (2002) 193-266.

[73] O.M. Philips, The entrainment interface, J. Fluid Mech. 51 (1972) 97-118.

[74] P.F. Embid, A.J. Majda, P.E. Souganidis, Comparison of turbulent flame speeds from complete averaging and the G-equation, Phys. Fluids 7 (1995) 2052-2060.

[75] B. Denet, Possible role of temporal correlations in the bending of turbulent flame velocity, Combust. Theory Modelling 3 (1999) 585-589.

[76] W.T. Ashurst, Flow-frequency effect upon Huygens front propagation, Combust. Theory Modelling 4 (2000) 99-106.

[77] B. Khouider, A. Bourlioux, A.J. Majda, Parameterizing the burning speed enhancement by smallscale periodic flows: I. Unsteady shears, flame residence time and bending, Combust. Theory Modelling 5 (2001) 295-318.

[78] M. Chinappi, M. Cencini, A. Vulpiani, Thin front propagation in random shear flows, Phys. Rev. E 73 (2006) 016308.

[79] A. Amato, T.C. Lieuwen, Analysis of flamelet leading point dynamics in an inhomogeneous flow, Combust. Flame 161 (2014) 1337-1347.

[80] L. Cifuentes, C. Dopazo, J. Martin, P. Domingo, L. Vervisch, Effects of the local flow topologies upon the structure of a premixed methane-air turbulent jet flames, Flow Turbulence Combust. 96 (2016) 535-546.

[81] T. Echekki, M.G. Mungal, Flame speed measurements at the tip of a slot burner: effects of flame curvature and hydrodynamic stretch, Symp. (Int.) Combust. 23 (1990) 455-464.

[82] N. Peters, Turbulent combustion, University Press, Cambridge, UK, 2000.

[83] V. Akkerman, V. Bychkov, Velocity of weakly turbulent flames of finite thickness, Combust Theory Modelling 9 (2005) 323-351.

[84] V. Bychkov, A. Petchenko, V. Akkerman, On the theory of turbulent flame velocity, Combust. Sci. Technol. 179 (2007) 137-151.

[85] J.-M. Truffaut, G. Searby, Experimental study of the Darrieus-Landau instability on an inverted-V flame, and measurement of the Markstein number, Combust. Sci. Technol. 149 (1999) 35-52. 